The Canadian Society for Clinical Investigation and the Clinician Investigator Trainee Association of Canada acknowledges the support of:

Canadian Institutes of Health Research Friends of CIHR

Bristol-Myers Squibb Canada Eli Lilly Canada Inc.

University of Ottawa

Burroughs Welcome Fund

\section{CSCI/CITAC Young Investigators Forum Abstracts}

CSCI/CITAC YOUNG INVESTIGATORS FORUM

for

CLINICAL SCIENTISTS IN TRAINING

CIP and MD/PhD STUDENTS

TRIBUNE DES JEUNES CHERCHEURS

pour

\section{CHERCHEURS CLINICIENS EN FORMATION ÉTUDIANTS INSCRITS AU PROGRAMMES PFCC ET MD/PHD}

Thursday, September 20, 2012

Jeudi, le 20 septembre 2012

The National Art Centre, Ottawa.

Ottawa, Ontario 


\section{1-01 Two year progression of subcortical grey matter iron accumulation in multiple sclerosis using high field MRI}

\section{Andrew Walsh, BSc ${ }^{1}$; Gregg Blevins, $\mathrm{MD}^{2}$; Alan Wilman, $P h D^{1}$}

${ }^{1}$ Department of Biomedical Engineering,

2 Division of Neurology, Faculty of Medicine and Dentistry, University of Alberta, Edmonton, Alberta, CANADA

Background: Iron has been implicated in the pathophysiology of multiple sclerosis (MS) and could be a marker of disease activity or contribute to disease progression [1]. Different MRI methods are sensitive to iron and have shown differences in deep grey matter nuclei between MS patients and healthy controls [2]. However the time evolution of iron accumulation is unclear. This study evaluates iron accumulation in deep grey matter over two years measured with quantitative MRI in early relapsing remitting MS (RRMS) patients compared to age matched controls.

Methods: Quantitative MRI using gradient echo R2* mapping was performed at with a 4.7T MRI system. Seven patients with early RRMS (age $35.2+/-5.5$, average disease duration $2.5+/$ 1.4 years) and seven healthy controls (age $35.0+/-9.6$ ) were imaged twice, each two years apart. Two-dimensional region of interest analysis was performed on deep grey matter nuclei including: caudate, globus pallidus, putamen, thalamus, pulvinar thalamus, substantia nigra, and red nucleus. Repeated measures ANOVA was used to determine temporal differences in $\mathrm{R} 2 *$ measurements.

Results: Substantia nigra $(\mathrm{p}=0.008)$ and red nucleus $(\mathrm{p}=0.028)$ showed significant interactions over time between patient/ control groups with a larger difference at the later time point. Putamen, thalamus, pulvinar thalamus, substantia nigra and red nucleus all showed a significantly higher value in RRMS compared to control subjects as a between subjects effect $(\mathrm{p}<0.05)$.

Conclusion: Since most deep grey matter structures showed differences in $\mathrm{R} 2{ }^{*}$ measures averaged over 2 years between RRMS patients and controls, it appears that there is an overall increase in deep grey matter iron in early RRMS. However, these changes take place before 2.5 years disease duration on average and represent very early iron changes while only two structures showed further change during the study period. It seems iron accumulation could take place at different rates in different deep grey structures in MS. More longitudinal studies, with multiple time points and various disease severity, are needed to clearly establish the temporal relationship between iron accumulation in specific deep grey matter structures in MS. The preliminary results indicate that iron changes occur during a short time scale in some structures in early RRMS, therefore quantitative R2* MRI measurements could be a valuable adjunct for diagnosis or prognosis.

\section{References:}

1. Williams R, JNC 2012

2. Lebel R, MS 2011

\section{1-02 Obstructive Sleep Apnea Treatment Improves Renin Angiotensin System Activity in Humans}

Nicholl DDM ${ }^{1}$; Hanly PJ'; Handley GB' ${ }^{1}$; Hemmelgarn BR ${ }^{1}$; Poulin MJ'; Sola DY'; and Abmed SB ${ }^{1}$

1 University of Calgary, Alberta, CANADA

Background: Obstructive sleep apnea (OSA) is strongly associated with cardiovascular (CV) and kidney disease. Limited studies suggest a prominent role of the renin angiotensin system (RAS), activation of which is deleterious to cardiorenal function. We sought to determine the effect of continuous positive airway pressure (CPAP) therapy in patients with OSA on mean arterial pressure (MAP) and RAS components at baseline and in response to Angiotensin II (AngII) infusion.

Methods: Ten newly diagnosed ( 8 men, 2 post-menopausal

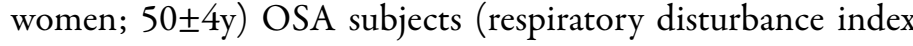
$[\mathrm{RDI}]>15$ ) with nocturnal hypoxia (oxyhemoglobin saturation [SaO2] $<90 \%$ for $>12 \%$ of night) who were otherwise healthy were studied pre- and post-CPAP therapy ( 1 month of adequate therapy [ $>4 \mathrm{~h} / \mathrm{night}])$. Subjects were studied in high salt balance, a state of maximal RAS suppression. MAP, plasma renin activity (PRA), and aldosterone were measured during each study period at baseline and in response to a graded AngII infusion $(3 \mathrm{ng} / \mathrm{kg} / \mathrm{minx} 30 \mathrm{~min}, 6 \mathrm{ng} / \mathrm{kg} / \mathrm{min} \times 30 \mathrm{~min}, 30 \mathrm{~min}$ recovery). MAP was measured every $15 \mathrm{~min}$. The primary outcome was the effect of CPAP therapy on MAP and RAS components at baseline and in response to AngII infusion.

Results: CPAP corrected OSA (RDI: $52 \pm 6$ vs $5 \pm 1 \mathrm{hr}-1$, $\mathrm{p}=0.005$; duration $\mathrm{SaO} 2<90 \%$ : $33 \pm 5$ vs $5 \pm 3 \%$ of night, $\mathrm{p}=0.005)$ and reduced baseline MAP $(97 \pm 1$ vs $89 \pm 2 \mathrm{mmHg}$, $\mathrm{p}=0.007)$, PRA $(0.27 \pm 0.02$ vs $0.19 \pm 0.03 \mathrm{ng} / \mathrm{L} / \mathrm{s}, \mathrm{p}=0.02)$, and aldosterone $(195 \pm 30$ vs $122 \pm 16 \mathrm{pmol} / \mathrm{L}, \mathrm{p}=0.009)$, but did 
not affect AngII (20 \pm 4 vs $20 \pm 4 n g / L, p=0.7)$. CPAP increased MAP sensitivity to $3 \mathrm{ng} / \mathrm{kg} / \mathrm{min}$ AngII ( $15 \mathrm{~min}: 10 \pm 2$ vs $16 \pm 2 \mathrm{mmHg}, \mathrm{p}=0.01$; $30 \mathrm{~min}: 12 \pm 3$ vs $15 \pm 3 \mathrm{mmHg}, \mathrm{p}=0.1$ ), but did not affect the MAP response to $6 \mathrm{ng} / \mathrm{kg} / \mathrm{min}$ AngII or recovery. There was a blunted PRA response post-CPAP (3ng/ $\mathrm{kg} / \mathrm{min}:-0.10 \pm 0.02$ vs $-0.07 \pm 0.02 \mathrm{ng} / \mathrm{L} / \mathrm{s}, \mathrm{p}=0.097 ; 6 \mathrm{ng} / \mathrm{kg} /$ min: $-0.17 \pm 0.02$ vs $-0.12 \pm 0.03 \mathrm{ng} / \mathrm{L} / \mathrm{s}, \mathrm{p}=0.05)$, but improved recovery $(-0.14 \pm 0.02$ vs $-0.09 \pm 0.03 \mathrm{ng} / \mathrm{L} / \mathrm{s}, \mathrm{p}=0.038)$.There were no changes in the aldosterone response to AngII.

Conclusions: CPAP treatment resulted in decreased RAS activity and increased hemodynamic sensitivity to AngII, supporting a role for the RAS in mediating OSA-induced hypertension. This is important given the increasing prevalence of OSA and high impact on CV morbidity/mortality, quality of life and health care costs.

\section{1-03 Macrophages mediate islet dysfunction in re- sponse to human islet amyloid polypeptide}

\section{Andrew Walsh, BSc ${ }^{1}$; Gregg Blevins, $\mathrm{MD}^{2}$; Alan Wilman, $P h D^{1}$}

${ }^{1}$ Department of Biomedical Engineering, ${ }^{2}$ Division of Neurology, Faculty of Medicine and Dentistry, University of Alberta, Edmonton, Alberta, CANADA

Background: Aggregation of human islet amyloid polypeptide (IAPP) to form amyloid fibrils contributes to beta cell dysfunction in type 2 diabetes and islet transplant failure. IAPP aggregates are present within islet macrophages and share a common cross beta-sheet structure with other amyloids that induce a potent pro-inflammatory response via activation of Toll-like receptor 2 (TLR2). We sought to evaluate the contribution of islet macrophages to IAPP-induced islet inflammation and beta cell dysfunction.

Methods: Bone marrow-derived macrophages (BMDMs) and isolated islets from wild-type and Tlr2-/- mice were treated with human IAPP $(10 \mu \mathrm{M})$ or non-amyloidogenic rodent IAPP and assayed for pro-inflammatory cytokines by qRTPCR and ELISA. The contribution of resident macrophages to IAPP-induced islet gene expression was assessed following phagocyte depletion with clodronate-containing liposomes. To determine the role of macrophages in IAPP-induced islet dysfunction in vivo, wild-type or human IAPP-expressing FVB mice were treated with clodronate liposomes $(100 \mathrm{mg} / \mathrm{kg}$ i.p. every 4 days) or PBS control liposomes beginning at 20 weeks of age. Glucose tolerance and islet gene expression were assessed after 4 weeks.

Results: Human but not rodent IAPP induced expression of the pro-inflammatory cytokines IL-1 $\beta$ ( $4.5 \pm 1.2$ fold untreated; $\mathrm{p}<0.05), \quad$ TNF- $\alpha \quad(10.3 \pm 0.7 ; \mathrm{p}<0.001), \quad$ IL-6 $(117 \pm 56$; $\mathrm{p}<0.001)$, and CCL2 $(10.2 \pm 0.6 ; \mathrm{p}<0.001)$ in islets after $4 \mathrm{~h}$. Clodronate-mediated macrophage depletion attenuated IAPPinduced pro-inflammatory cytokine expression. Similarly, in vivo macrophage depletion prevented the 4 -fold increase in $\mathrm{CD} 11 \mathrm{~b}+$ cell infiltration associated with human IAPP expression, blocked IAPP-induced islet IL-1 $\beta$ mRNA expression $(0.16 \pm 0.05$ vs. $1.9 \pm 0.3$ fold wild-type littermate control, $\mathrm{p}<0.001)$, and improved IAPP-induced glucose intolerance. Upregulation of IL1 $\beta$, TNF- $\alpha$, IL- 6 , and CCL 2 was attenuated in Tlr2-/- islets, suggesting that TLR2 signalling is required for the pro-inflammatory response to IAPP.

Conclusions: These data suggest that islet macrophages are the major source of IAPP-induced pro-inflammatory cytokines, and that diverse amyloidogenic peptides act as endogenous stimuli for TLR2. Thus, manipulation of macrophage activation or blockade of TLR2 may limit IAPP-induced islet inflammation and beta cell dysfunction in type 2 diabetes and islet transplantation.

Financial Support: CWR is funded by a Vancouver Coastal Health-CIHR-UBC MD/PhD Studentship and a Vanier Canada Graduate Scholarship.

\section{1-04 The Valproic Acid-Fludarabine combination in-} duces a synergistic response in Chronic Lymphocytic Leukaemia via a mechanism involving the lysosomal protease Cathepsin B

Clara Y. Westwell-Roper ${ }^{1,2}$; Jan A. Ehses ${ }^{3}$; C. Bruce Verchere ${ }^{1,3}$ ${ }^{1}$ Department of Pathology and Laboratory Medicine, University of British Columbia

${ }^{2} \mathrm{MD} / \mathrm{PhD}$ Program, University of British Columbia

${ }^{3}$ Department of Surgery, University of British Columbia

Background/Purpose: Chronic Lymphocytic Leukaemia (CLL) is the most common haematological malignancy in the western world. Fludarabine, a nucleoside analogue, is commonly used to treat CLL in untreated and relapsed CLL. However, patients commonly develop resistance to fludarabine. We hypothesize that the addition of Valproic Acid (VPA), an 
inhibitor of histone deacetylases (HDACs), can improve fludarabine-based therapy.

Methods: The pharmacodynamic relationship between VPA and fludarabine was examined in vitro in human leukaemic cell lines and primary CLL cells. Protein levels were examined by immunoblotting. Apoptosis was quantified by examining for cells with hypo-diploid DNA content by flow cytometry. Cathepsin B activity was measured by combining cell lysates with the fluorogenic substrate zRR-AMC. Chart review was performed to assess the results of the phase II clinical trial.

Results: The VPA-Fludarabine combination induced a synergistic response in human leukaemic cells and primary CLL cells. Combined treatment induced activation of caspases-2, -8, -9 and caspase-3. Enhanced activation of caspases was associated with down-regulation of two prominent anti-apoptotic proteins, Mcl-1 and XIAP. The down-regulation of Mcl-1 and XIAP was dependent on the lysosomes, as their alkalinization using either chloroquine or $\mathrm{NH} 4 \mathrm{Cl}$ partially stabilized both proteins, leading to reduced apoptosis. Furthermore, chemical inhibition of a specific lysosomal protease, cathepsin B, using CA074-Me, also reduced apoptosis in response to VPAfludarabine. Addition of purified cathepsin B to leukaemic cell lysates was sufficient to induce degradation of both Mcl-1 and XIAP in vitro. VPA treatment enhanced cathepsin B levels and its activity, as assessed by the cleavage of zRR-AMC. In the clinic, six relapsed CLL patients who had received at least one prior therapy with fludarabine were examined. No responses were seen after 28 days using VPA alone. However, in five patients who continued on VPA with fludarabine, 3 patients showed a $>50 \%$ fall in lymphocyte/lymph node size after receiving 5 cycles of the combination.

Conclusions: A novel mechanism was discovered through which VPA interacts with fludarabine synergistically by enhancing the activity of cathepsin $\mathrm{B}$. The resulting enhancement in caspase activation was associated with reduction in $\mathrm{Mcl}-1$ and XIAP levels. The combination was also effective in relapsed CLL patients.
1-05 Transplantation of adult- and embryonic stem cell-derived neural precursor cells preserves tissue and improves neurobehavioural function in a rat contusioncompression model of cervical spinal cord injury

Wilcox JT ${ }^{1,2}$; Satkunendrarajah $\mathrm{K}^{2}$; Nasirzadeb $\mathrm{Y}^{2}$; Nassiri

F1, Lip $A^{2}$; Fehlings $M G^{1,2}$

${ }^{1}$ Institute of Medical Science, University of Toronto, and ${ }^{2} \mathrm{Di}-$ vision of Genetics and Development, Toronto Western Research Institute, University Health Network, Toronto, Ontario, CANADA

Introduction: Thoracic rodent models of spinal cord injury (SCI) predominate as preclinical translational models; however, cervical SCI presents as the majority of clinical cases and represents the most significant personal impact and societal burden.

Methods: The applicability and utility of thoracic and lower cervical SCI preclinical models was evaluated using clip contusion-compression injury paradigm in rats. Spinal injuries were realized in female Wistar rats at the C5, C6, C7 and T7 vertebral levels (controls: laminaectomy-only) using severe clip contusion-compression ( $28 \mathrm{~g}$ for $60 \mathrm{~s}$ ). Animals were assessed for 10 weeks using: grip strength meter, Inclined Plane test, WARP contracture scale, BBB locomotor scale, bladder recovery, motor- and somatosensory-evoked potentials (MEP, SEP), and $\mathrm{H}$ reflex. LFB/H\&E histological staining and T1weighted MR imaging were used to assess tissue loss and cavitation. Adult- and embryonic stem cell-derived neural precursor cells (aNPC and ES-NPC, respectively) were transplanted $\left(4 \times 10^{\wedge} 5\right.$ cells, or vehicle only) into C6-injured rats at 2 weeks post-injury and followed with similar neurobehavioural assessment.

Results: Spontaneous neurological recovery was observed with expected plateaus in all injury groups. Numerous behavioural and electrophysiological testing modalities demonstrated that hindlimbs and autonomic function were similar to all injury levels (BBB, BBB subscore, bladder recovery and MEP, $\mathrm{p}>0.05)$. Conversely, injury to $\mathrm{C} 5$ and $\mathrm{C} 6$ levels show marked forelimb and throacoappendicular motor deficits (grip and IP, $\mathrm{p}<0.001 ; \mathrm{H} / \mathrm{M}$ spasticity ratio and WARP, $\mathrm{p}<0.05$ ).

T1-weighted MR revealed similar and overt signal changes in all injuries, as confirmed with quantitative lesion analysis using LFB/H\&E. Following aNPC transplantation in $\mathrm{C} 6$ animals, grip strength increased 2-fold compared to vehicle controls $(417+/-59 \mathrm{~g}$ vs. $211+/-55 \mathrm{~g}, \mathrm{p}=0.007)$, while Inclined 
Plane and BBB showed no difference $(\mathrm{p}=0.15$ and 0.88 , respectively). Transplantation of aNPC and ES-NPC improved spared white and grey matter $(\mathrm{p}<0.05)$ compared to control groups. Electrophysiology also revealed significant improvements to lower peak amplitude and latency in SEP of forelimbs

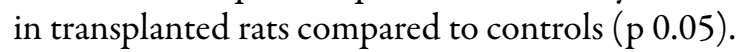

Conclusion: Injury at the mid-to-high cervical (C5, C6 vertebral level) is needed in models designed to study cervical SCI. The C6 clip paradigm appropriately models neurobehavioural recovery and spasticity of cervical SCI, which is ameliorated in tissue preservation and neurobehavioural function to the forelimb following adult and ES-derived NPC transplantation.

\section{1-06 A novel synthetic GMCSF and IL7 fusion cyto- kine (GIFT7) leads $T$ cell neogenesis by reversing age- related thymic atrophy and overcoming PD-1 - assocaited CD8 exhaustion}

\section{Jeremy Hsieb ${ }^{1,3}$; Colleen McGary ${ }^{2}$; Mirko Paiairdini², Lucca Micci²; Guido Silvestri' ${ }^{2}$; Jacques Galipeau ${ }^{3}$}

${ }^{1}$ Montreal Center for Experimental Therapeutics in Cancer, Lady Davis Institute, McGill University, Montreal, Canada ${ }^{2}$ Yerkes National Primate Research Center, Emory University, Atlanta, USA 3. The Winship Cancer Institute, Emory University, Atlanta, USA

Background: The competence of cellular immunity depends on a functional $\mathrm{T}$ cell pool with diverse $\mathrm{T}$ cell receptor (TCR) repertoire. Numerous cytotoxic assaults exist to perturb effector cell fitness. On the one hand, irradiation, chemotherapy, virus, and ageing are all capable of limiting de novo $T$ cell production from the thymus; and on the other hand, malignancies and chronic viral infection predispose peripheral CD8 T cells to exhaustion. Thymc involution and PD-1 upregulation remain the most flagrant expression of such immune dysfunction, which represents a huge challenge to the clinical management of affected population.

Methods: To address this issue, we report the development of a novel synthetic fusion cytokine arisig from the partnering of Granulocyte-macrophage colony stimulating factor (GMCSF) and Interleukin 7 Fusion Transgene (GIFT7).

Results: Thymus is the site of post-natal T cell development. In vitro, we demonstrate that GIFT7 promtes the proliferation of thymoctyes, particularly the CD4-CD8-CD25-CD24CD44+ (DN1) subset.

In vivo, systemic administration of GIFT7 in young mice leads to a potent increase in DN1 after 7 days and a reversible expansion of total thymocytes after 14 days. In aged mice $(>12$ months), GIFT7 significantly expands CD44intCD25- DN progenitors, which leads to an increase in total thymic cellularity and hyperplasia in the cortex where early $\mathrm{T}$ cell precursors seed after migrating from the bone marrow. GIFT7-induced thymopoiesis also enhanced anti-CMV T cell response.

Furthermore, we found that in the periphery, GIFT7 selectively expand a CD8+ subset with a Central Memory (CM) phenotype defined as CD8+CD44+CD62L+CCR7+KLRG$\mathrm{CD} 27+\mathrm{PD} 1-$. The unique expansive effect of GIFT7 is also demonstrated on peripheral blood mononuclear cells (PBMC) derived from non-human primates (NHP), in that GIFT7 stimulation of pre-activated NHP-PBMC leads to significant $\mathrm{T}$ cell proliferation without the upregulation of PD1.

Conclusion: Hence, we propose the use of GIFT7 or GIFT7primed $\mathrm{T}$ lineage cells for the treatment of immune malfunction.

\section{1-07 Cytoband with genes necessary for pathogenesis of fast-onset mouse plasmacytoma shows altered nuclear localization}

Kuzyk, $A .{ }^{1}$; Mai, $S .^{1}$

${ }^{1}$ University of Manitoba

Background/Purpose: A characteristic of fast-onset mouse plasmacytomas (PCTs) is trisomy of cytoband 11E2. This cytoband is known to harbor all the genes necessary for the accelerated tumorigenesis of fast-onset PCT. As the human synteny region to mouse cytoband 11E2 (17q25) is also duplicated, translocated and amplified in many lymphoid and nonlymphoid tumors, this region may harbor an oncogenic cluster important in tumor pathogenesis. To further study this cytoband, tumors were induced in $[\mathrm{T} 38 \mathrm{HxBALB} / \mathrm{c}] \mathrm{N}$ mice with a reciprocal translocation between chromosomes (chrs) $\mathrm{X}$ and $11(\operatorname{rcpT}(\mathrm{X} ; 11))$. This offers a unique opportunity to study cytoband $11 \mathrm{E} 2$ because the $\operatorname{rcpT}(\mathrm{X} ; 11)$ generates a very small $\mathrm{T}(\mathrm{X} ; 11)$ translocation chr that contains only cytoband 11E2. The nuclear localization of genes in the nucleus is thought to alter their rate of transcription, commonly with higher rates towards the center of the nucleus. Therefore we determined the 
$3 \mathrm{D}$ nuclear localization of this cytoband and the genes required for fast-onset PCT tumorigensis.

Methods: Primary B cells were harvested from wild-type $[\mathrm{T} 38 \mathrm{HxBALB} / \mathrm{c}] \mathrm{N}$ mice, with and without $\operatorname{rcpT}(\mathrm{X} ; 11)$, and from fast-onset PCTs. Fluorescence in situ hybridizations were conducted with chr-specific paints which label chrs X and 11 in $2 \mathrm{D}$ metaphases and $3 \mathrm{D}$ interphase nuclei. After highresolution imaging and constrained iterative deconvolution, the nuclear positions of labeled regions were quantitatively determined.

Results: The nuclear localization of chrs $\mathrm{X}$ and 11 is significantly different between the wild-type mice, with or without $\operatorname{rcpT}(X ; 11)$. Also, the position of chr $X$ and 11 in the same cell is significantly different. Metaphases from fast-onset PCTs reveal that trisomy of $11 \mathrm{E} 2$ can occur through duplication of chr 11 or $\mathrm{T}(\mathrm{X} ; 11)$ or an intrachromosomal duplication of the $11 \mathrm{E} 2$ band. The nuclear localization of $\mathrm{T}(\mathrm{X} ; 11)$ varies, possibly depending on the method of 11E2 duplication, with one copy of 11E2 always in the center of nucleus. The position of $\mathrm{T}(\mathrm{X} ; 11)$ significantly different between the wild-type and fastonset PCT mice.

Conclusion: The nuclear localization of chromosomes $\mathrm{X}$ and 11 are non-random and cell-type specific. Cytoband 11E2, which contains the genes necessary for fast-onset PCT tumorigenesis, has an altered nuclear localization compared to wildtype mice.

\section{2-01 The development of an in vitro cell culture model to study the pregnant human uterus}

\section{Andrea A. Mosher', Seunghwa S. Bolstad ${ }^{1}$, Kelly J. Rainey', Stephen Wood ${ }^{2}$, Donna M. Slater ${ }^{1,2}$}

1Physiology \& Pharmacology, ${ }^{2}$ Obstetrics \& Gynaecology, Faculty of Medicine, University of Calgary, Calgary, Alberta, Canada

Background/Purpose: Little is known about the cellular mechanisms involved in the initiation of labour. Preterm labour is a major problem in obstetrics. The development of in vitro cell culture models has greatly facilitated our ability to study human systems. However, there are concerns about the lifespan of cultured cells and whether they remain representative of the tissue of origin. The myometrium is the contractile machinery of the uterus, which is composed of smooth muscle cells and undergoes significant growth and remodelling throughout pregnancy. The objective of this study was to establish primary cultured human myometrial cells that could be used to study the cellular mechanisms involved in labour and to examine the expression of smooth muscle markers and fibroblast markers in these cells.

Methods: Myometrial biopsies were obtained from women undergoing Caesarean section at term, prior to labour onset ( $n=4 ; 38-40$ weeks gestation). Paired biopsies were taken from both the upper segment and the lower segment of the uterus. Myometrial cells were isolated from the biopsies and cultured to confluence. Cells were passaged every 72 hours and plated at a density of $4.0 \times 104$ cells $/ \mathrm{ml}$. To assess the response to a cytokine known to be involved in labour, cells were stimulated with IL-1 $\beta(1 \mathrm{ng} / \mathrm{ml})$. This treatment was repeated from passage 1 (p1) to p10. RT-PCR and immunocytochemistry were used to identify the smooth muscle markers $\alpha$ smooth muscle actin, calponin, caldesmon, and tropomyosin and the fibroblast markers vimentin and $1 \mathrm{~B} 10$.

Results: We demonstrate that both upper and lower segment human myometrial cells stably express smooth muscle markers and fibroblast markers until at least p10, suggesting that these cells represent myofibroblasts. Both cell populations retain their ability to respond to IL-1 $\beta$, demonstrated by a robust release of CXCL8 (20 000-50 $000 \mathrm{pg} / \mathrm{ml}$ ) and increased mRNA expression of COX-2. There were no significant differences in gene expression from p1-p10 for any of the genes studied.

Conclusions: Primary human myometrial cells can be isolated from myometrial biopsies and cultured to study the human uterus. These cells retain their ability to respond to an inflammatory stimulus for at least ten passages and exhibit a myofibroblast phenotype, demonstrated by expression of both smooth muscle markers and fibroblast markers. This cell culture model will greatly enhance our ability to study critical mechanisms involved in the onset of labour. 
2-02 Recognizing uncertainty in your knowledge: The impact of response options presentation on selfmonitoring accuracy.

Cynthia Min 1,2,3, Kevin W.Eva ${ }^{2,3}$

${ }^{1} \mathrm{MD} / \mathrm{PhD}$ Program, ${ }^{2}$ Faculty of Education, ${ }^{3}$ Centre for Health Education Scholarship (CHES), dDepartment of Medicine, Faculty of Medicine, The University of British Columbia

Background: To optimize patient care, physicians are expected to have a "moment-to-moment" awareness of the likelihood of being correct. This has been termed self-monitoring and occurs when physicians recognize uncertainty in their clinical decision making and stop to seek additional information. It has been argued that self-monitoring is crucial in clinical practice because it carries the most immediate impact on patient safety. A series of studies using short answer questions have demonstrated a relationship between self-monitoring and response accuracy. The current study used multiple-choice questions to determine if eliminating the need to generate a response would impact the accuracy of self-monitoring.

Methods: 40 participants were randomly allocated to answer 60 multiple-choice questions in which response options were presented before or after participants had to decide whether or not to answer. For each question, they were asked to answer if they were confident and to defer otherwise. All deferred questions were then required to be answered on a second presentation. Measures of self-monitoring included accuracy of attempted versus deferred questions and the speed at which such decisions were made.

Results: Respondents showed indications of accurate selfmonitoring in both experimental conditions. When response options were provided prior to deciding to answer or defer respondents answered attempted questions with $52.05 \%$ accuracy and deferred questions with $27.65 \%$ accuracy. This difference was equivalent when the decision to answer or defer was made prior to seeing the response questions $(52.40 \%$ vs $34.05 \%$, respectively). For both conditions, questions answered on first presentation had a faster response time when correctly answered compared to when questions incorrectly. In both conditions, deferred questions that were correctly answered had a slower decision time compared to incorrectly answered questions.

Conclusion: The findings indicate that the accuracy of selfmonitoring was not influenced by the opportunity to review possible responses prior to judging the likelihood of being able to provide the correct answer. Furthermore, the results suggest a relationship between response time the accuracy of responses. Further explorations should be undertaken to determine whether self-monitoring is a teachable skill that can aid clinical decision making.

Financial Support: C.M. is supported by a CHES-CIHRUBC MD/PhD Studentship Award and a UBC 4-Year Fellowship Award.

\section{2-03 Antipsychotic drugs promote the development of oligodendrocyte through transcription factors olig1 and olig2 in vitro}

\section{Yanbo Zhang ${ }^{1,2}$; Handi Zhang ${ }^{2}$; Junhui Wang ${ }^{2}$; Shenghua}

$\mathrm{Zhu}^{2}$; Jue He${ }^{2}$; Jiming Kong ${ }^{3}$; Xin-Min Li ${ }^{1,2,3}$

${ }^{1}$ Department of Psychiatry, College of Medicine, University of Saskatchewan, Saskatoon, SK, CAN

2Department of Psychiatry, Faculty of Medicine, University of Manitoba, Winnipeg, MB, CAN

3Department of Human Anatomy and Cell Science, University of Manitoba, Winnipeg, MB, CAN

Objective: Neuroimaging and microarray studies have indicated that oligodendrocyte and myelin abnormalities are important pathological changes of schizophrenia. Antipsychotic drugs (APDs) are effective in treatment of schizophrenia; but the underlying mechanism remains unknown. Our previous studies suggested that quetiapine, an atypical antipsychotic drug, promoted neural progenitor cells to differentiate into oligodendrocyte lineage cells and alleviated CPZ-induced demyelinating pathology. In this project, we further investigated the effect of different antipsychotic drugs on the oligodendrocyte in vitro.

Methods: A well-established oligodendrocyte-lineage cell line, CG4 cells were used to exam the effect of three antipsychotic drugs: haloperidol, quetiapine and olanzapine. Antipsychotics showed no effects on proliferation of CG4 cells evaluated by CCK-8 proliferation assay. However, all of the drugs promoted differentiation of CG4 cells into mature oligodendrocytes when it was evaluated by the expression of CNPase, a maker of mature oligodendrocyte. Further to investigate the mechanism of antipsychotic drugs, we found the expression of oligodendrocyte transcription factor 1 (olig1) and 2 (olig2) were distinctly regulated by the drugs. 
Results: The expression of olig2 was up-regulated by the all the drugs tested and olig1 was only increased by quetiapine and olanzapine, but not by haloperidol. These data suggested that olig1 and olig2 may play a key role in the regulation process of APDs on oligodendrocyte development and there may be some differences between the action of typical and atypical antipsychotics.

Conclusions: Our results indicate APDs have effects to promote the differentiation of CG4 oligodendrocyte cell line in vitro and oligodendrocyte/myelin may be a novel target for APDs.

\section{2-04 Community-acquired pneumonia hospitalizations and HIV in the Saskatoon Health Region}

Olfert, Jordan'; Skinner, Stuart ${ }^{2}$

${ }^{1}$ Department of Medicine and 2 Department of Infectious Diseases, University of Saskatchewan, Saskatoon, SK, CAN

Background: There has been an outbreak of HIV in the Saskatoon Health Region (SHR) since 2005. Thirty patients have been diagnosed in hospital since 2008, and the majority diagnosed late in disease with missed opportunities for earlier diagnosis. Community-acquired pneumonia (CAP) is a common presentation in patients with HIV. We suspect that HIV is now the leading underlying co-morbidity in young adults admitted with CAP in SHR. Testing for HIV in these patients may result in earlier diagnosis.

Methods: A retrospective chart review of 230 patient admissions, aged 18-50 years, with CAP to SHR hospitals in 2010. 110 admissions satisfied all inclusion and exclusion criteria. The presence of underlying co-morbidities, including a previous or new diagnosis of HIV, was determined.

Results: HIV was the leading co-morbidity, comprising 28 (25\%) patient admissions. Five (4.5\%) patients were newly diagnosed with HIV. Four newly diagnosed patients fulfilled criteria for AIDS, of which one patient was diagnosed postmortem.

Conclusion: The Centers for Disease Control and Prevention recommend screening for HIV in all patients aged 13-64 years in all healthcare settings, unless the prevalence of those undiagnosed is $<0.1 \%$. This study provides evidence for standard testing of HIV in all patients aged $18-50$ years admitted with CAP. The testing requirement of $0.1 \%$ was far exceeded by the value of $4.5 \%$ obtained in this study. Considering those newly diagnosed were in advanced stages of infection, further methods must be explored to diagnose these patients earlier on in the course of infection.

\section{2-05 Prophylactic Nasal CPAP in the Post-Anaesthesia Care Unit Following Elective Laparotomy}

Hoeppner $E^{1}$; Ong $D^{1}$; McKay $W^{1}$

${ }^{1}$ University of Saskatchewan, Department of Anaesthesiology

Background: The objective of this study was to determine the effect on pulmonary gas exchange of nasal continuous positive airway pressure (nCPAP) following elective laparotomy for bowel surgery when compared to oxygen delivered by a simple face mask. Abdominal surgery is a risk factor for post-operative pulmonary complications (PPCs) such as hypoxemia, pneumonia and respiratory failure.[1] PPCs lead to increased mortality and hospital length of stay.[2] CPAP is a method of respiratory support that decreases atelectasis, work of breathing and hypoxemia. $[3,4]$ The effect of prophylactic nCPAP on gas exchange following laparotomy for bowel surgery has not been evaluated.

Methods: This study was a prospective, randomized controlled trial. Patients scheduled to undergo elective laparotomy for bowel surgery were included. Patients who were less than 18 years of age or were admitted to the intensive care unit postoperatively were excluded. Upon arrival to the post-anaesthesia care unit (PACU), thirty-seven subjects were randomized by opening opaque envelopes containing computer generated randomization numbers corresponding to the control group or the study group. The control group received oxygen at 8 litres per minute $(\mathrm{lpm})$ through a simple face mask. The study group received $\mathrm{nCPAP}$ at $10 \mathrm{~cm} \mathrm{H} 2 \mathrm{O}$ plus oxygen at $81 \mathrm{pm}$. An arterial blood gas was obtained after one hour of treatment to measure the partial pressure of arterial oxygen $(\mathrm{PaO} 2)$ and the partial pressure of arterial carbon dioxide ( $\mathrm{PaCO} 2)$. Pulmonary gas exchanged was measured using the alveolar to arterial oxygen gradient (A-aDO2).

Results: The median (standard deviation) $\mathrm{A}-\mathrm{aDO} 2$ in the nCPAP group was 70.0 (43.9) $\mathrm{mmHg}$ vs. the control group which was $140.5(71.9) \mathrm{mmHg}(\mathrm{P}<0.01)$. The results for age, gender, ASA status, smoking history, narcotic use and presence of an epidural were similar $(\mathrm{P}>0.05)$. The control group had a higher body mass index (BMI) of 30.5 (6.7) vs. 26.7 (5.8) $(\mathrm{P}=0.05)$ and fewer males, 6 vs. $13(\mathrm{P}=0.03)$. 
Discussion: The results show that prophylactic $\mathrm{nCPAP}$ at $10 \mathrm{~cm}$ $\mathrm{H} 20$ for one hour following elective laparotomy for bowel surgery decreases the A-aDO2 when compared to oxygen delivered at $81 \mathrm{pm}$ by a simple face mask. This indicates that prophylactic nCPAP, a method of non-invasive respiratory support, improves post-operative pulmonary gas exchange. Elevated BMI has been associated with increased atelectasis formation.5 The results may be partly explained by the BMI difference between the two groups.

\section{References}

1. Ann Intern Med. 2006;144:581-595.

2. J Am Coll Surg. 2004;199(4):541-7.

3. Chest. 1987;92:621-624.

4. Am J Respir Crit Care Med. 1997;155:500-505.

5. Anesth Analg. 2002;95(6): 1788-92.

\section{2-06 Osteoarthritis develops in an anatomically ideal ovine ACL autograft}

Huebner KD; O'Brien EJO; Beveridge JE; Heard BJ, Tapper JE, Shrive NG; Frank CB

McCaig Institute for Bone and Joint Health, Faculty of Medicine, University of Calgary. 3330 Hospital Drive NW, Calgary, AB. CAN

Background/Purpose: The development of osteoarthritis (OA) following anterior cruciate ligament (ACL) injury and reconstruction is a clinical problem occurring in $\sim 60 \%$ of patients. The research suggests that normal mechanics and joint stability may not be fully restored post-reconstruction, possibly explaining the incidence of OA. New surgical techniques have attempted to mimic a more anatomic approach; however, have failed to reduce the occurrence of OA. Our aims were to evaluate functional joint stability in an idealized ACL reconstruction model. We hypothesized that there would be no alteration to gait mechanics and no gross evidence of OA $20 \mathrm{wks}$ postsurgery compared with sham surgery and non-surgical sheep.

Methods: Five mature female sheep underwent unilateral idealized ACL reconstruction (ACL-R), in which the native ACL is cored out at the femoral end and immediately replaced in its native position. Seven animals served as shams and seventeen animals served as non-operated controls. The gait cycle of the ACL-R were compared with shams using $3 \mathrm{D}$ motion analysis at 4 and 20 wks post-operatively. Gross joint changes were assessed and graded at the time of euthanasia.
Results: Of the 48 points of the gait cycle compared, 42 points showed no difference between ALC-R and Shams. The 6 points of significant difference were distributed across 4 degrees of freedom with $3 / 6$ significant changes seen in rotation. More specifically, ACL-R had greater abduction at $20 \mathrm{wks}$ and greater internal rotation at 4 and 20 wks. ACL-R also showed significantly increased anterior tibial translation at 4 wks and superior translation at 20 wks. There was no difference in differential gross morphology scores at 20 wks between shams and non-operated controls, while ACL-R joints had a significantly higher combined score than controls. Inter-animal differences were noted in all groups.

Conclusion: This model simulates a number of features of human ACL reconstructive surgery and eliminates some confounding factors such as variation in graft attachment site and the use of non-ligamentous graft material. However, we were unable to restore normal gait kinematics and prevent the development of OA with this method of ACL reconstruction in sheep. Further work is necessary to confirm and identify the association between the kinematic abnormalities and the initiation of OA in this model, and tease out biological contributors to disease.

\section{2-07 Regional gradient of Blood Oxygen Level De-} pendant Cardiovascular MR (BOLD-CMR) in patients with Coronary Artery Disease: A comparative study with Fractional Flow Reserve.

\section{Judy Luu, MD/PhDc ${ }^{2}$; Jacqueline Flewitt, M.Sc. ${ }^{1}$; Matthias G. Friedrich, MD. ${ }^{1}$ \\ ${ }^{1}$ CMR Centre at the Montreal Heart Institute, Montreal, Quebec; ${ }^{2}$ Stephenson CMR Centre at the Libin Cardiovascu- lar Institute of Alberta.}

Background: BOLD-CMR exploits the inherent magnetic property of hemoglobin with the signal intensity (SI) in T2*weighted images being dependent on its oxygenation status. The technique has been used to detect myocardial ischemia in patients with coronary artery disease (CAD). As ischemic myocardial damage occurs primarily in the subendocardium, we hypothesized that there is a transmural oxygenation gradient in patients with CAD.

Methods: Patients scheduled for clinically-indicated coronary angiography $(\mathrm{n}=18$, mean age $60.6 \pm 9.9$ yrs, 15 male) and 
healthy controls $(n=11$, mean age $29 \pm 4$ yrs, 6 male) were scanned using a clinical 1.5T MRI system. BOLD-CMR was performed at rest and during adenosine-induced vasodilation in 3 short-axis slices in the patient group and a single midventricular slice for healthy volunteers. Change in BOLD signal intensity (SI) from rest to adenosine was calculated for the sub-epicardial and -endocaridal regions. In patients, segmental analysis was compared with the fractional flow reserve (FFR) and quantitative coronary angiography (QCA). An FFR cutoff of 0.80 was used to define significant coronary artery stenosis.

Results: All images from the healthy volunteers were used. In 18 patients, 81 segments with accompanying FFRs and 125 segments with accompanying QCA were analyzed. CAD patients had an overall reduced response to hyperemia across the whole myocardium when compared to healthy subjects. There was no transmural gradient to the BOLD SI in healthy subjects, whereas CAD patients had significantly different responses in the sub-epicardium versus the sub-endocardium in all segments, regardless of the FFR. Response in segments subtended by coronaries with an abnormal FFR had even further reduced BOLD SI change upon hyperemia and an even more significant difference transmurally between sub-epicardium and sub-endocardium (Figure 1). There was no correlation between the sub-epicardial $(\mathrm{r}=-0.075, \mathrm{n}=125, \mathrm{p}=0.407)$ or subendocardial $(\mathrm{r}=0.041, \mathrm{n}=125, \mathrm{p}=0.647)$ BOLD SI change and percent stenosis as measured by QCA.

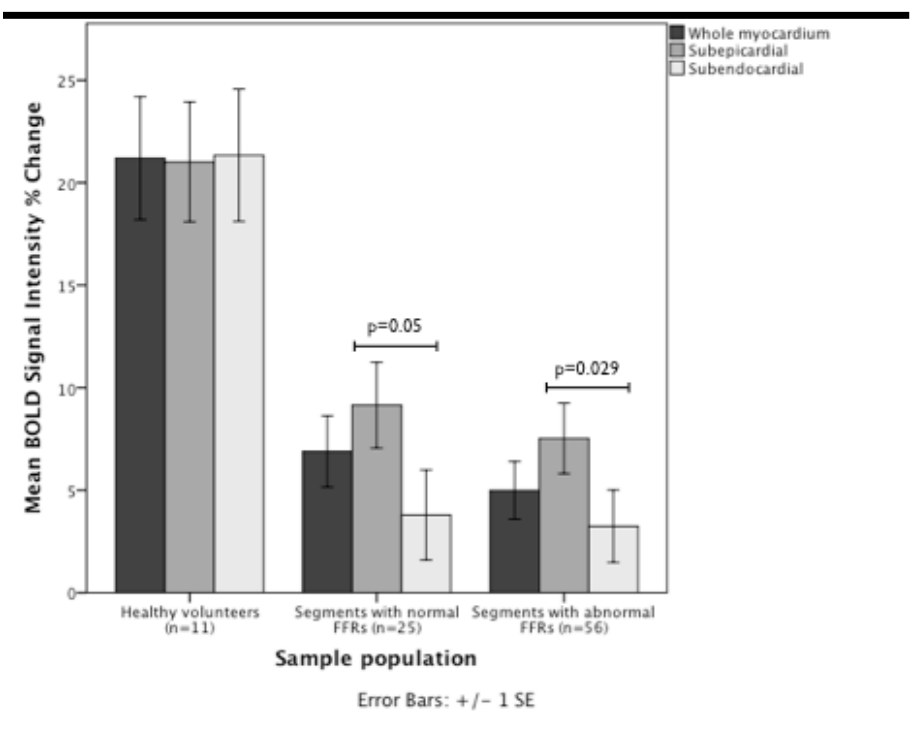

FIGURE 1 (2-07). Transmural changes in BOLD SI in healthy volunteers and $\mathrm{CAD}$ patients.
Conclusion: BOLD-CMR can distinguish regional changes in oxygenation present in CAD patients and not present in healthy subjects. Segments with abnormal FFR values were the furthest reduced and had the most significant difference transmurally. However, segments subtended by normal FFR values also exhibited these changes, demonstrating the presence of abnormal oxygenation despite a vessel determined by FFR to be free of significant stenosis. This technique provides a noninvasive transmural assessment of myocardial oxygenation and may prove to be a sensitive tool for detection of ischemia over the currently employed gold standards.

\section{2-08 Prenatal depression in Aboriginal women: The "Voices and PHACES" Study}

\section{Amrita Roy ${ }^{1}$, Wilfreda Thurston ${ }^{1,2}$, Scott Patten ${ }^{1,3}$, Suzanne Tough ${ }^{1,4}$, Lynden Crowshoe ${ }^{5}$, Tanya Beran ${ }^{1}$ \\ ${ }^{1}$ Dept of Community Health Science, ${ }^{3}$ Dept of Psychiatry, ${ }^{4}$ Dept of Pediatrics, ${ }^{5}$ Dept of Family Medicine (Faculty of Medicine); ${ }^{2}$ Dept of Ecosystem and Public Health (Faculty of Veterinary Medicine), University of Calgary, AL. CAN}

Background: Prenatal depression carries serious consequences for maternal and child health. Risk factors and health consequences associated with prenatal depression are more prevalent in Aboriginal populations, but the specific determinants in this population have not been well-studied. Given the unique historical and social contexts involved, we hypothesize that the determinants differ relative to non-Aboriginal populations. Greater knowledge will enable more effective intervention and prevention strategies.

Objectives: 1) To identify determinants of prenatal depression among Aboriginal women, and map out a theoretical framework; 2) To assess the adequacy and appropriateness of programs and services in Calgary for pregnant Aboriginal women.

Methods: Constructivist grounded theory methodology was employed, involving semi-structured interview guides and theoretical sampling of participants. Personal interviews were conducted with pregnant Aboriginal women in Calgary, and the health and social services professionals who work with them. The research was conducted in partnership with community organizations, and with the guidance of an Oversight Committee which included Aboriginal community members.

Results: Preliminary results from our ongoing study point to various determinants. These include risk factors consistent with 
other prenatal depression studies, including stressful life events, social and economic factors, negative previous pregnancy experiences, and experiences of violence and abuse. Unique factors identified in this study include experiences with the child welfare system, family members' residential school experiences, historical trauma, stigma, racism and sexism. Negative coping mechanisms include substance abuse. Social support and access to Aboriginal traditional practices appear protective. While services in Calgary are working well in certain ways, there is a need for more culturally-appropriate services, better networking among agencies, and more education of service-providers to reduce stigma. The reluctance of some women to link their experiences of unfair treatment with their wellbeing may reflect the normalization and internalization of oppression.

Significance: Understanding the unique determinants of depression that we are identifying among pregnant Aboriginal women can aid in the design of appropriate and effective interventions.

\section{2-09 The Role of Prenyl Diphosphate Synthase Subunit 1 (PDSS1) in Impaired Cellular Bioenergetics and its Implications in Obesity}

\author{
Ghadi Antoun ${ }^{1}$; Robert Dent ${ }^{2}$; Ruth McPherson ${ }^{1}$; Mary-Ellen \\ Harper ${ }^{1}$ \\ ${ }^{1}$ Department of Biochemistry, Microbiology and Immunology, \\ Faculty of Medicine, University of Ottawa and Ottawa Insti- \\ tute of Systems Biology \\ ${ }^{2}$ University of Ottawa Heart Institute
}

Background: According to the World Health Organization, obesity has more than doubled since 1980, affecting over 1.5 billion adults over the age of 20[1]. The 2008 Canadian statistics reveal that over 8 million people suffer from obesity, with estimated annual healthcare related costs exceeding $\$ 4.6$ billion[2]. Given that obesity is also a major risk factor for the development of other conditions such as cardiovascular diseases, diabetes, osteoarthritis, and some cancers (including endometrium, breast, and colon) $\mathrm{i}$, it is clear that it constitutes an important area of research. Advancements in the field, more specifically in elucidating the genetic predictors of obesity, could contribute significantly to its prevention and possible treatment.

Coenzyme Q10 (CoQ10), is a lipid-soluble component of cell membranes that plays an essential role in the mitochondrial electron transport chain (ETC) and consequently on cel- lular energy expenditure processes. Specific deficiencies of this biomolecule have been previously reported to lead to multisystem diseases including obesity[3]. Recently, Mollet et al. demonstrated that mutations in the Prenyl Diphosphate Synthase Subunit 1 (PDSS1) gene lead to CoQ10 deficiencies[4]. PDSS1 codes for an enzyme that dimerizes with PDSS2 to elongate the prenyl side chain, a key step in CoQ10 biosynthesis. Preliminary work from our skeletal muscle genome-wide association studies has also identified PDSS1 as an important genetic locus bearing significance to obesity and weight loss success.

Goal: The goal of this project is thus to evaluate the role of PDSS1 in mitochondrial bioenergetics, linking expression of this important gene to cellular energy expenditure, metabolism, and development of obesity. We hypothesize that decreased PDSS1 gene expression will lead to a reduction in CoQ10 levels. This will diminish aerobic respiration and impair energy expenditure processes leading to increased cellular energy storage and susceptibility to obesity.

Results: Given that skeletal muscle is responsible for $20 \%$ of whole-body resting metabolic rate, advances in understanding cellular energy expenditure processes in this tissue give us insight into whole-body bioenergetics and obesity development. Hence, cultured samples of these cells will serve as a model system for examining the effect of PDSS1 on skeletal muscle metabolism. Much of the work was conducted in a commonly used mouse $\mathrm{C} 2 \mathrm{C} 12$ myoblast cell line using an in vitro siRNA gene knockdown approach. Expected results include decreased levels of PDSS1 expression, which will lead to a reduction in CoQ10 levels, as well as modified cellular metabolism. Bioenergetic and physiological outcomes should be reversed by ubiquinone supplementation, suggesting that PDSS1 regulates metabolism through synthesis of CoQ10. Measurements of PDSS1 and CoQ10 expression levels in skeletal muscle of obese and lean individuals will also be carried out to extend our analyses into the clinical environment. In obese individuals, PDSS1 and CoQ10 levels will also be compared between distinct clinical populations exhibiting differential weight loss during a closely supervised clinical caloric restriction weight loss program at The Ottawa Hospital Weight Management Clinic.

Higher PDSS1 expression levels are expected in lean and obese diet-sensitive (ODS) compared to obese diet-resistant (ODR) patients both at the protein and mRNA levels. 
Conclusion: Generally, we expect that our findings will yield an improved understanding of the biochemical underpinnings of variable energetic efficiency characteristics and their roles in the development and treatment of obesity. Ultimately, we are hoping that our results will allow the development of novel therapeutic and pharmaceutical strategies to improve weight loss success in clinical programs.

\section{References:}

1. Obesity and overweight, Fact sheet $\mathrm{N}^{\circ} 311$, World Health Organization, Updated March 2011

(http://www.who.int/mediacentre/factsheets/fs311/en/)

2. Obesity in Canada, Overview, Public Health Agency of Canada, Updated June 2011

(http://www.phac-aspc.gc.ca/hp-ps/hl-mvs/oic-oac/index-eng.ph p)

3. Quinzii CM, López LC, Naini A, DiMauro S, Hirano M., Human CoQ10 deficiencies. Biofactors. 2008;32(1-4):113-8.

4. Mollet J, Giurgea I, Schlemmer D, Dallner G, Chretien D, Delahodde A, Bacq D, de Lonlay P, Munnich A, Rötig A. Prenyldiphosphate synthase, subunit 1 (PDSS1) and OH-benzoate polyprenyltransferase (COQ2) mutations in ubiquinone deficiency and oxidative phosphorylation disorders. J Clin Invest. 2007

Mar;117(3):765-72.

\section{2-10 miR-125b: A Potential OncomiR in Cutaneous Squamous Cell Carcinomas}

\section{Lorie M. Kwong; Jennifer M. Tran; Habib Kurwa; P. Régine Mydlarski}

Division of Dermatology, Department of Medicine, University of Calgary, Calgary, AB, CAN

Background/Purpose: Non-melanoma skin cancer (NMSC) is the most common form of cancer worldwide. While ultraviolet radiation is the primary risk factor for developing NMSC, much remains unknown about the process of skin carcinogenesis. miRNAs are small, non-coding RNAs that regulate gene expression. Certain miRNAs function as oncogenes or tumour suppressors, yet their roles in the promotion and progression of cancer are not clearly established. Herein, we investigate the role of miRNAs in cutaneous squamous cell carcinomas (SCC).

Methods: To compare the miRNA profile of normal human keratinocytes (NHK) and cutaneous SCC cells, total miRNA was extracted from commercially available cell lines. Using $\mu$ Paraflo ${ }^{\circ}$ Microfluidic Biochip technology and probe content based on the Sanger miRBase Version 12.0, a genome-wide miRNA microarray analysis was performed.

To examine the expression of miR-125b in SCC tumours, miRNA was isolated from patient-matched normal and SCC tissue pairs $(\mathrm{n}=4)$ and miR-125b expression was quantified through qRT-PCR techniques.

miR-125b was exogenously upregulated in SCC cells using synthetic miRNA mimics. The effects of miR-125b deregulation on cellular proliferation, differentiation, apoptosis and invasion were examined.

Results: Of 856 miRNAs studied, 27 miRNAs displayed high signal intensities and were significantly up- or down-regulated in the SCC cell lines. While the majority of miRNAs in the genome remain uncharacterized, miR-125b, a highly downregulated miRNA in SCC cells, has been implicated in the carcinogenesis of numerous cancers. miRNA analysis of matched normal skin and SCC tissues confirmed miR-125b suppression in all SCC tumours.

In vitro studies indicate miR-125b does not regulate cellular proliferation or apoptosis in SCCs. However, exogenous deregulation of miR-125b in SCCs induces a loss of cellular differentiation that results in a more invasive phenotype.

Conclusions: The identification of unique miRNA profiles within NHKs and SCCs allows our dataset to serve as a roadmap for future studies of the microRNAome. By characterizing the role of miR-125b in SCCs, molecular insight will be gained into the process of skin cancer development.

2-11 Ultra-fast two-photon microscope based on acousto-optic deflector for imaging of synaptic and neuronal morphology in brain of in vivo Xenopus Tadpole for study of schizophrenia

Philip Edgcumbe; Kasper Podgorski; Kurt Haas

University of British Columbia, BC, CAN

Background/Purpose: Many neurological disorders, including autism, epilepsy and schizophrenia are associated with abnormal early brain development. During early brain development neurons grow elaborate dendritic and axonal arbors that form precise inter-neuronal synaptic connections and functional neural circuits.

Methods: We have custom-built a random access ultra- fast two-photon microscope based on acousto-optic deflectors that 
generate $x-y$ images of $300 \times 300$ pixel resolution with diffraction limited resolution of $600 \times 600 \mathrm{~nm}$ per pixel. A piezoelectric focusing element controls the position of the objective along the optical axis (z-direction) and allows for whole neuron imaging at a rate of $100 \mathrm{hz}$. We built the ultra-fast two photon microscope so we can study schizophrenia in the in vivo model system of the Xenopus tadpole. To do this we will simultaneously image the synaptic and morphological plasticity in vivo in single tectal neurons of Xenopus tadpoles. This allows us to study how schizophrenia affects synaptic plasticity, the correlation between synaptic firing and the relationship between synaptic plasticity and morphological plasticity. It is important to understand if seizures that occur during development affect essential processes associated with neural circuit formation.

We have created a novel visual stimulation imaging chamber for the Xenopus Laevus tadpole. The calcium-sensitive fluorescent indicator Oregon green 488 BAPTA-1 was bulk loaded into neurons within the tadpole brain and light stimuli were presented to the tadpoles and the response of the tadpoles neuronal network was recorded.

Results: Visual stimulation elicited a significant neuronal network response in the optic tectum of the Xenopus tadpole with an increase in Oregon green 488 BAPTA intensity of up to $10 \%$ in regions of interest. Preliminary visual stimulation results, discussion of future research and design of ultra-fast twophoton microscope are presented.

Conclusion: Our custom-built ultra-fast two-photon microscope, novel custom-built visual stimulation imaging chamber and associated preliminary visual stimulation results form the basis of a new experimental platform for studying early brain development.

Finical Support: Philip Edgcumbe is supported by a Vancouver Coastal Health-CIHR-UBC MD/PhD Studentship Award.

\section{2-12 Preconditionning of stem cells by oxytocin to im- prove their therapeutical potential in the treatment of cardiovascular disease}

Alexandra Desnoyers; Mélanie Borie; Abmed Menaouar;

Marek Jankowski; Jolanta Gutkowska; Denis Claude Roy;

Samer Mansour; Louis Mathieu Stevens; Nicolas Noiseux

Centre de Recherche du Centre Hospitalier de l'Université de Montréal (CRCHUM) and Université de Montréal, Montréal, QC, CAN

Background/Purpose: The objective is to evaluate a novel strategy of preconditioning mesenchymal stem cells (MSC) with oxytocin (OT) to enhance their therapeutical potential. Our hypothesis is that treatment of MSC with OT will activate important cell signalling pathways improving survival and function of transplanted cells in ischemic conditions such as myocardial infarction.

Methods: Rat MSC (rMSC) have been used in these experiments and treated with OT 10-6 to 10-10 M. The cellular response of the signalling pathways PI3K/Akt and ERK1/2 ( $\mathrm{p} 44 / \mathrm{p} 42$ ), and gene expression were assessed by Western blots and real-time PCR. In vitro migration of MSC was measured with a modified Boyden chamber. Effects on viability, cell cycle and apoptosis were evaluated by BrdU, TUNEL and flow cytometry.

Results: A short stimulation with OT induces a rapid phosphorylation (activation) of Akt (2X) and p44/p42 (ERK1/2; $17 \mathrm{X})$. These pathways are involved in various cellular functions such as proliferation, differentiation, motility, survival and intracellular trafficking. OT significantly increased cell proliferation by up to $78.0 \%$ at 72 hrs compared with non-stimulated cells. PI flow cytometry demonstrated an increase of $8.32 \%$ in $S$ phase of the cell cycle when rMSC were stimulated with OT. Cell viability in a serum-free medium and in hypoxic conditions was significantly improved by OT, this increase was blocked by the inhibitors of ERK (PD98059) and Akt (wortmannin) pathways as well as by the antagonist of OT receptor, demonstrating their involvement. The TUNEL analysis of serum starved rMSC demonstrated a protective effect of OT with a reduction of apoptosis by $40 \%$ in normoxic and $73 \%$ in hypoxic conditions respectively. Migration of rMSC preconditioned with OT was significantly increased up to $69 \%$, maximum with OT10-6M. OT positively modulates gene expression of antiapoptotic and angiogenic proteins: VEGF, TIMP1, MMP-2 and TGFb1. In addition, expression of cytoprotec- 
tive genes (heat shock proteins, HSPs) were increased: HSP27, HO-1 (HSP32) and HSP70.

Conclusion: In this study, the combination of simple pharmacological treatment to cell therapy represents a novel strategy to improve effector cell profile, which can be applied to various cell types prior to transplantation, limiting potential systemic effects and without genetic modification. With stem cell conditioning, we may eventually harness the therapeutic potential of these unique cells to better fulfill the beneficial promise of regenerative medicine.

\section{2-13 TYPE and Prevalence of Clinical Information Provided on Chest Radiograph Requisitions}

\section{Guan Huang MD ${ }^{1}$; Navneet Singh MD $^{2}$; Alan Moody} FRCR $^{2}$; Klaus K Gast MD ${ }^{2}$

${ }^{1}$ Department of Radiology and Diagnostic Imaging, University of Alberta

${ }^{2}$ Department of Medical Imaging, University of Toronto

Background: Clinicians may receive reports with broad differential diagnostic considerations by radiologists requiring "clinical correlation". Provided clinical history improves the rate of true positive readings [1-3]. One potential contributing factor of indefinitive readings may be the lack of clinical information available to the interpreting radiologist. Thus, this quality assurance investigation sought to review the prevalence and type of clinical history provided.

Methods: The local institutional ethics review board approved this chart review study. Of the chest radiographs requested by ED physicians at SBHSC in August and September of 2011, $\mathrm{n}=100$ without prior chest radiographs were randomly selected. There were 54 female and 46 male patients with an age range of 1 to 100 and a median age of 74 . Clinical information was categorized into "history", "physical exam findings", "lab values" and "to rule out certain disease".

Results: A total of 201 pieces of clinical information were identified, with 135 (67 \%) in "history", 15 (8\%) in "physical exam findings", 4 (2\%) in "lab values" and 48 (23\%) in "to rule out certain disease". Every chest radiograph was supplied with at least one piece of clinical information. 45, 26, 15, 11 and 3 chest radiographs were provided with 1, 2, 3, 4 and 5 pieces of information, respectively.
Conclusion: Limited clinical history is provided on radiographic requisitions of the chest from the emergency department, with nearly half of the requisitions providing only one piece of clinical information. Physical exam findings and lab values were seldom provided. Further clinical information may allow the radiologist to narrow diagnostic possibilities to assist the referring clinician in the management of their patients.

\section{References:}

1. Schreiber $\mathrm{MH}$. The clinical history as a factor in roentgenognam interpretation. JAMA 1963; 185: 137-139.

2. Potchen EJ, Gard JW, Lazan P, Lahaie P, Andany M. The effect of clinical history data on chest film interpretation: direction or distraction. Invest Radiol 1979;i4 : 404.

3. Doubilet P, Herman PG. Interpretation of radiographs: effect of clinical history. AJR Am J Roentgenol. 1981;137:1055-1058.

\section{2-14 Treatment of a Multi-Ligament Injured Knee: A Case Report of Bicruciate Peel-Off Lesions}

Graeme Matthewson; David Simon; Gregory Stranges

Leaders In Medicine Programme, University of Calgary, Calgary, $\mathrm{AB}, \mathrm{CAN}$

Department of Orthopedic Surgery, Pan Am Clinic, Faculty of Medicine, University of Manitoba, Winnipeg, MB

Background: Multiple ligament injured knee's (MLIK's) are a rather rare but potentially fatal traumatic event, with $32 \%$ of cases involving vascular and 20-30\% involving neurological complications. We report here, a case of a 35 year old female soccer player who sustained a multi-ligamentous injury to her right knee during play.

Purpose: To investigate a case of MLIK with double primary repairs of the anterior cruciate ligament (ACL) and posterior cruciate ligament (PCL) as this combination has yet to be reported in the literature.

Methods: The MLIK was arthroscopically repaired with a primary ACL repair to the tibial spine and a primary PLC repair to the femur, using the native ligaments in both. A Tibialis Anterior allograft was used for reconstruction augment of the posterolateral corner using the Larson technique. This procedure employed the use of a button suture to secure the PLC (biceps femoris, fibular collateral ligament and posterolateral capsule) to the fibular head. 
Results: Post surgery, a standard rehabilitation program was implemented with induction of range of motion exercises at 46 weeks. At two weeks post procedure, on $\mathrm{x}$-ray there was pull out of the suture button from the fibular head. At 10 weeks post procedure the patient had reduced range of motion, which was suspected to be due to arthrofibrosis of the knee. After 22 weeks of diligent physiotherapy and rehabilitation the patient returned for follow up. An MRI was done approximately 30 weeks after her initial surgery, which showed evidence of popliteal muscle atrophy with an intact tendon and intact cruciate and collateral ligaments. However, the anterior central knee was suspect for arthrofibrosis, which further supported the clinical suspicion at 10 weeks.

Discussion: The most serious long-term complication with any MLIK is stiffness. One of the main barriers of returning to sport is pain and swelling, and the development of an arthrofibrotic joint. Recently, there have been methods employed to reduce the degree of arthrofibrosis including delaying treatment to greater then 1 week post injury, aggressive rehabilitation and also unicruciate vs bicruciate repair.

Conclusion: Primary repair of bicruciate tears in a MLIK should implement the same principles for reduction of arthrofibrosis as has been developed in bicruciate reconstruction.

\section{2-15 Contrast Enhanced US in Inflammatory Bowel Disease: Should it be an Occasional or an Essential Component of Every Exam?}

Aman Wadhwani ${ }^{1}$, Alexandra Medellin ${ }^{1}$, Kerri L. Novak ${ }^{2}$ Stephanie R. Wilson ${ }^{1,2}$

${ }^{1}$ Department of Diagnostic Imaging, ${ }^{2}$ Division of Gastroenterology, Faculty of Medicine, University of Calgary, Calgary, $\mathrm{AB}$

Background/Purpose: A clinical challenge in managing Crohn disease $(C D)$ is establishing reliable, non-invasive methods to assess disease activity. Increasingly, this is performed on traditional ultrasound(tUS) with greyscale assessment of bowel wall thickening, inflammatory fat and adenopathy. Color Doppler imaging(CDI) allows subjective assessment of blood flow, as a reflection of inflammation. Our purpose is to show the benefit to this assessment of the addition of the recently introduced technique of contrast enhanced ultrasound (CEUS) of the bowel. Where available, CEUS activity prediction is compared with gold standard colonoscopy.
Method: CDI, tUS and CEUS were performed on $100 \mathrm{CD}$ patients. All scans were reviewed by 2-blinded readers to determine CD activity on the baseline scan with CDI. Then, disease activity was objectively assessed on CEUS to show its impact on the initial assessment, upgrading or downgrading disease activity from the initial predictions based on tUS with CDI.

Results: 60 patients showed concordance between tUS and CDI, 36/60 of which showed either moderate or severe inflammation and 24/60 showed quiscent or mild inflammation of the bowel wall. In the indeterminate group of 40 patients, CDI did not corroborate tUS findings, generally showing thick bowel wall without expected blood flow on CDI. Therefore, disease activity could not be confidently predicted in the indeterminate group on the baseline scan alone. The addition of CEUS upgraded disease activity (none/mild/moderate/severe) in $4 / 60$ of the patients belonging to the concordant group. However, the disease activity was upgraded in 22/40 of patients belonging to the indeterminate group. Downgrading with CEUS was infrequent in both groups. In the 36 patients with concordant result of baseline US and CDI suggesting moderate to severe disease, CEUS did not suggest significantly different disease activity. The overall confidence in predicting disease activity in the indeterminate group was low. However, using CEUS increased reader confidence level by $58-64 \%$ with an overwhelming majority in the indeterminate group. Limited numbers of analyzed colonoscopies show a clear trend in peak enhancement between milder and more severely inflamed bowel.

Conclusion: CEUS is optimally used to determine the disease activity in IBD patients on whom baseline prediction with CDI and tUS is indeterminate. Performing CEUS selectively on CD patients may better predict the disease activity in a noninvasive manner with optimal use of resources.

\section{2-16 Rural Trauma Systems - Demographic \& Out- come Analysis of Remote North Quebec Traumas}

\section{Fadi Hamadani}

McGill University Health Centre, Montreal, QC, CAN

Objective: Studies have shown mortality \& morbidity rates from traumatic injury to be higher in remote $\&$ rural populations compared to urban areas. In remote North Quebec com- 
munities, transport to the Montreal University Health Centre (MUHC), a level-1 trauma centre, is the only option for complex trauma care. This study aims to provide: (1) a demographic analysis of the Northern Quebec region, with an emphasis on characterizing the available health care infrastructure; (2) the mechanisms \& rates of injuries in the North that require transfer; (3) a comparison of outcomes, for the same injury severity score (ISS), between patients arriving directly to the MUHC and those requiring transport from the North.

Methods: A retrospective analysis of all trauma patients with an injury severity score of 16 or more that were entered into the MUHC trauma registry from Jan 2005-December 2009 was performed, identifying patients transported from the North separately. Data collected from both groups was analyzed to derive frequencies, means and percentages for the various parameters, which were then used to calculate the standardized mortality ratio (observed / predicted) for both populations.. A student's t-test was used for continuous and Chi-squared test was used for discontinuous data. The level of significance was set at $\mathrm{P}<0.05$.

Results: A demographic assessment of available health services in North Quebec was performed (Table 1). There were 3772 local traumas with ISS $>16$ compared to 172 that arrived from North Quebec. The mean age for both groups was $>40$ years with a predominantly male population, the majority of which had sustained blunt trauma. MVC was the most common mechanism in both groups, averaging $40 \%$ for both populations. Penetrating trauma was the cause of $12 \%$ of all transports from the North, whereas it represented $7 \%$ of the injuries seen in the local population. Table 2 illustrates the outcomes parameters. There was no significant difference in unadjusted mortality rate between the two groups, but the standardized mortality ratio was higher in the Northern group.

TABLE 1 (2-16): Questionnaire items and respective responses for each of the questionnaire items by Staff Physicians

\begin{tabular}{|c|c|c|c|c|c|c|c|c|}
\hline Question & No & N/A & Never & $\begin{array}{l}\text { Almost } \\
\text { Never }\end{array}$ & $\begin{array}{c}\text { Some- } \\
\text { times }\end{array}$ & $\begin{array}{l}\text { Almost } \\
\text { Always }\end{array}$ & Always & Yes \\
\hline $\begin{array}{l}\text { How often do you use seat belts in the car when you } \\
\text { drive or ride in a car? }\end{array}$ & & $0 \%$ & $0 \%$ & $0 \%$ & $0 \%$ & $5 \%$ & $95 \%$ & \\
\hline $\begin{array}{l}\text { How often do you exceed the posted speed limit } \\
\text { when you drive? }\end{array}$ & & $0 \%$ & $1 \%$ & $14 \%$ & $65 \%$ & $18 \%$ & $2 \%$ & \\
\hline $\begin{array}{l}\text { During the past } 30 \text { days, have you driven when } \\
\text { you've had too much to drink? }\end{array}$ & $97 \%$ & & & & & & & $3 \%$ \\
\hline $\begin{array}{l}\text { During the past } 30 \text { days, have you ridden with a } \\
\text { driver who has had too much to drink? }\end{array}$ & $96 \%$ & & & & & & & $4 \%$ \\
\hline $\begin{array}{l}\text { When you rode a bicycle during the past year, how } \\
\text { often did you wear a helmet? }\end{array}$ & & $31 \%$ & $6 \%$ & $0 \%$ & $4 \%$ & $7 \%$ & $52 \%$ & \\
\hline $\begin{array}{l}\text { When you played a contact sport during the past } \\
\text { month, how often did you wear the complete rec- } \\
\text { ommended protective gear? }\end{array}$ & & $84 \%$ & $0 \%$ & $0 \%$ & $3 \%$ & $3 \%$ & $10 \%$ & \\
\hline $\begin{array}{l}\text { How often do you cross the road anywhere else } \\
\text { other than an intersection? }\end{array}$ & & $0 \%$ & $0 \%$ & $19 \%$ & $73 \%$ & $8 \%$ & $0 \%$ & \\
\hline How often do you wear a life-jacket while boating? & & $13 \%$ & $2 \%$ & $10 \%$ & $15 \%$ & $19 \%$ & $41 \%$ & \\
\hline $\begin{array}{l}\text { How often do you check the depth of the water you } \\
\text { are about to dive into before diving? }\end{array}$ & & $30 \%$ & $0 \%$ & $1 \%$ & $2 \%$ & $9 \%$ & $58 \%$ & \\
\hline Do you have a working smoke detector in the home? & $0 \%$ & & & & & & & $100 \%$ \\
\hline Do you have a working CO detector in the home? & $17 \%$ & & & & & & & $83 \%$ \\
\hline $\begin{array}{l}\text { Are there any working revolvers, pistols, or hand- } \\
\text { guns in your home? }\end{array}$ & $100 \%$ & & & & & & & $0 \%$ \\
\hline $\begin{array}{l}\text { I always wear a helmet when riding an ATV or off- } \\
\text { road vehicle. }\end{array}$ & & $92 \%$ & $1 \%$ & $0 \%$ & $0 \%$ & $0 \%$ & $7 \%$ & \\
\hline I always wear a helmet when riding a motorcycle. & & $89 \%$ & $1 \%$ & $0 \%$ & $0 \%$ & $0 \%$ & $10 \%$ & \\
\hline
\end{tabular}


TABLE 2 (2-16): Patient Outcomes for both populations.

\begin{tabular}{|c|c|c|c|}
\hline \multirow{2}{*}{ Patient Outcome } & \multicolumn{2}{|c|}{ Mean $(\%)$} & \\
\hline & Local & North & p-value \\
\hline Mortality during treatment & $6.7 \%$ & $8.5 \%$ & 0.464 \\
\hline Standardized mortality ratio & 2.2 & 4.12 & $0.036^{*}$ \\
\hline
\end{tabular}

Conclusion: The study is a novel comparison between two different trauma populations. Despite longer transfer times in the Northern population, unadjusted mortality rates were the same amongst both groups.

\section{2-17 Surgical landmarks of the fronto-temporal branch of the facial nerve: A three dimensional study.}

Davies, J.C.; Fattah, A.; Ravichandiran, M.; Agur, A. Division of Anatomy, Department of Surgery, University of Toronto, Toronto, ON, CAN

Backgroud: The fronto-temporal branch of the facial nerve (FTN) is vulnerable to injury during craniofacial surgeries due to its superficial course and variable distribution (Gosain et al., 1997). Surface landmarks of the face that correlate with the underlying course of the FTN can assist in surgical planning. Current estimates of the course of the FTN commonly rely on Pitanguy's line, which utilizes soft-tissue landmarks that are variable between individuals. Location of important anatomic structures pre-operatively should focus on bony landmarks that are fixed, constant and easily palpable. Although the course of the FTN has been described based on peri-operative images, line drawings and manual measurement, no study has proposed surface landmarks for identification of the FTN using 3D analysis.

Purpose: To evaluate the effectiveness of using palpable surface landmarks to predict the course and distribution of the FTN using $3 \mathrm{D}$ modeling.

Methods: Surface bony landmarks including the porion, supraorbital notch, inferior orbital margin, supraorbitomeatal line (SOML) and infraorbitomeatal line (IOML) were demarcated and digitized using a Microscribe digitizer in ten cadaveric formalin embalmed specimens. A preauricular flap was raised using a modified Blair incision and branches of the FTN were isolated and digitized using a retrograde dissection to the origin of the nerve trunk. The digitized data were reconstructed into 3D models using Maya.
Results: In contrast to previous findings, the FTN was observed to divide into a variable number of branches and was distributed throughout the temporal region. The most posterior branch of the FTN was $19 \pm 3 \mathrm{~mm}$ and $18 \pm 4 \mathrm{~mm}$ from the porion along the SOML and IOML, respectively. The most anterior branch of the FTN was $62 \pm 5 \mathrm{~mm}$ from the supraorbital notch along the SOML and $56 \pm 4 \mathrm{~mm}$ from the inferior orbital margin along the IOML.

Conclusions: Digitization and three-dimensional modeling provides a unique method of visualizing the course and quantifying the distribution of the FTN in relation to surface bony landmarks. The data may be used in pre-operative planning to minimize injury to the FTN during craniofacial surgeries.

2-18 A 3D study of the musculotendinous architecture of piriformis and the sciatic nerve using in situ digitization: a fresh look at piriformis syndrome

David N. Parente'; Shannon L. Roberts ${ }^{1}$; Oonagh H. Scallan $^{1}$; Philip A. Fabrizio ${ }^{2}$; F. Richard Clemente ${ }^{3}$; Anne M. Agur ${ }^{1}$

${ }^{1}$ Division of Anatomy, Department of Surgery, University of Toronto, Toronto, ON M5S 1A8, Canada. ${ }^{2}$ Department of Physical Therapy, College of Pharmacy and Health Sciences, Mercer University, Atlanta, GA 30341, USA. ${ }^{3}$ Department of Physical Therapy, John G. Rangos Sr. School of Health Sciences, Duquesne University, Pittsburgh, PA 15282, USA.

Background/Purpose: Compression of the sciatic nerve (SN) as it exits the pelvis through the greater sciatic foramen has been implicated as a potential cause of sciatica or piriformis syndrome. However, the anatomical foundation of this claim has not been studied in sufficient detail to support the mechanism. The purpose of this study is to document the musculotendinous architecture of piriformis and its relationship with the greater sciatic foramen, and $\mathrm{SN}$.

Methods: In seven formalin-embalmed cadaveric specimens, obturator internus, gemelli, and the greater sciatic foramen were digitized using a Microscribe ${ }^{\oplus}$ G2X Digitizer. Piriformis was serially dissected, digitized, and modeled in 3D using Autodesk ${ }^{\circ}$ Maya. The muscle bundle architecture and extent of internal aponeurosis was analyzed and related to the position of the $\mathrm{SN}$ and greater sciatic foramen. 
Results: Medially, the aponeurosis is intramuscular and as it courses laterally to converge with the tendon of insertion, becomes superficial on the anterior surface of piriformis. The aponeurosis was found to have an average width of $22.6 \mathrm{~mm}$ and length of $76.3 \mathrm{~mm}$. The superficial portion of the aponeurosis coursed an average of $60.7 \mathrm{~mm}$ before becoming intramuscular. The $\mathrm{SN}$ was observed to be in direct contact with the superficial aponeurosis of piriformis and the bony ischial spine in all 7 specimens leaving the $\mathrm{SN}$ in a vulnerable position as it exits the pelvis.

Conclusion: Three-dimensional modeling of the position of the $\mathrm{SN}$ in relation to the musculotendinous architecture of piriformis allows for the evaluation of the feasibility of compression at this site. Future studies should be directed towards evaluation of these findings using in vivo imaging of patients with suspected sciatic pain due to piriformis syndrome.

\section{2-19 Variations in the course of the sacral nerve roots} relative to the fiber bundle architecture of piriformis and their potential clinical implications: a threedimensional modeling study.

\section{David N. Parente ${ }^{1}$; Shannon L. Roberts'; Oonagh H. Scal- lan $^{1}$; Philip A. Fabrizio ${ }^{2}$; F. Richard Clemente ${ }^{3}$; Anne M. Agur $^{1}$}

${ }^{1}$ Division of Anatomy, Department of Surgery, University of Toronto, Toronto, ON M5S 1A8, Canada. ${ }^{2}$ Department of Physical Therapy, College of Pharmacy and Health Sciences, Mercer University, Atlanta, GA 30341, USA. ${ }^{3}$ Department of Physical Therapy, John G. Rangos Sr. School of Health Sciences, Duquesne University, Pittsburgh, PA 15282, USA.

Background/Purpose: Intramuscular sacral nerve root compression by the piriformis muscle (PM) has been hypothesized as a possible cause of sciatica. However, the relationship of the sacral nerve roots to the PM has not been well documented. The purpose of this ongoing study is to visualize and quantify the position of the sacral nerve roots as they relate to the fiber bundle architecture of the PM.

Methods: In seven formalin-embalmed cadaveric specimens, the anterior rami of the L4-S3 nerve roots and communicating branches that form the sciatic nerve $(\mathrm{SN})$ were digitized along with the volume of the PM using a MicroScribe ${ }^{\circ}$ G2X Digitizer and were reconstructed in 3D using Autodesk ${ }^{\circ}$ Maya. The path of each nerve root was traced from the anterior sacral foramina to the $\mathrm{SN}$ and defined relative to the fiber bundle architecture of the PM. The presence of intra- and extramuscular divisions was recorded and characterized.

Results: The anterior rami of L4, L5, S1, and S3 were extramuscular in all specimens, traveling on the anterior surface of the $\mathrm{PM}$, whereas $\mathrm{S} 2$ was intramuscular in three of seven specimens. The $\$ 2$ nerve root in two specimens was covered anteriorly by muscular slips of the PM and, in one specimen, had a significant intramuscular course, which modified its trajectory. The angle that was created in $S 2$ was measured using three points: i) the point of emergence from the anterior sacral foramen, ii) the point of emergence from its intramuscular course in the PM, and iii) the point of its contribution to the SN. This marked angle was found to be $154^{\circ}$, compared to an average angle of descent of $175^{\circ}$ in the other specimens.

Conclusion: The course of the S2 nerve root may be deviated by fiber bundles of the PM lying anterior to its emergence from the anterior sacral foramen by mechanical forces of muscle contraction. Angulation of $S 2$ at the point it becomes extramuscular may render it susceptible to being stretched by contraction of the PM, manifesting as radicular pain. These findings need to be further investigated using in vivo imaging studies in patients with suspected piriformis syndrome.

\section{2-20 Intramuscular Compartmentalization of the Subscapularis Based on Neuromuscular Innervation Patterns}

Chang ${ }^{1}$ Youjin; Julia Warden ${ }^{2}$; Shannon Roberts ${ }^{1}$; Ross Baker ; Amila Samarakoon "; Chris Boulias ${ }^{2}$; Farooq Ismail'; Anne Agur ${ }^{1}$

${ }^{1}$ Divisions of Anatomy and ${ }^{2}$ Physiatry, Faculty of Medicine, University of Toronto, Toronto, ON, CAN

Background: Subscapularis is an important component of the rotator cuff. The infraclavicular nerves including the subscapular nerves are at risk of injury following open shoulder repair resulting in denervation of the muscle. Although variability has been found in the extramuscular innervation pattern, the knowledge on the intramuscular innervation is currently lacking.

Purpose: The study aims to explore extra- and intramuscular innervation patterns of subscapularis using cadaveric dissection and 3D modelling. 
Methods: All branches entering the muscle belly were identified and digitized in seven formalin-embalmed cadaveric specimens. Each intramuscular branch was digitized throughout the muscle volume and reconstructed into a 3D model using Maya. The model was used to determine neuromuscular partitioning patterns within subscapularis. An additional 23 specimens were then dissected to study extramuscular variations.

Results: Four intramuscular innervation patterns were observed based on the number of primary extramuscular branches (range: 2-5) to subscapularis. Subscapularis was then neuromuscularlly partitioned into two (superior and inferior) or three (superior, middle, and inferior) parts based on intramuscular innervation pattern.

Conclusion: Innervation of subscapularis is highly variable, with the number of primary branches ranging from two to five, originating from the posterior cord, axillary nerve, or suprascapular nerve. Based on intramuscular nerve distribution patterns, subscapularis can be partitioned into two or three parts. The proportion of muscle volume denervated by extramuscular nerve lesions may be directly related to intramuscular compartmentalization, which varies by innervation pattern.

\section{2-21 Genomic Imprinting in the Preimplantation Em- bryo: The Effects of Rate of Embryo Development}

\section{B. A. Market Velker; M. M. Denomme; M. R.W. Mann}

Children's Health Research Institute and the Department of Obstetrics and Gynecology, University of Western Ontario, Schulich School of Medicine and Dentistry, London, ON, CAN

Background: One of the first observations suggesting deleterious effects of in vitro embryo culture was an 18-24 hour lag in mouse preimplantation development. While culture conditions have steadily improved, even the best media are suboptimal. Currently, stage of embryo development has been proposed as one of many criteria for identifying "healthy" embryos in IVF clinics, with "fastest" embryos highlighted as "healthiest". However the validity of this as accurate criteria with respect to genomic imprinting is unknown. We have previously demonstrated that genomic imprinting, an epigenetic phenomenon where expression is dictated by parental origin, is disrupted during preimplantation embryo culture. Given the culture-induced delay in development, we hypothesize that loss of imprinting correlates with slower rates of embryonic development.

Methods: To evaluate this, embryos were recovered at the 2-cell stage, separated into four groups based on morphological stage at two predetermined time points, and cultured to the blastocyst stage. We examined cell number, embryo volume, embryo sex, imprinted methylation and expression at two key loci, H19 and Snrpn, known to be involved in the development of imprinting disorders observed in the ART population, imprinted expression of the cell cycle inhibitor, Cdkn1c, to explore the connection between cell cycle and genomic imprinting, and expression of three genes involved in embryo metabolism.

Results: We observed that fastest developing embryos exhibited the greatest cell number and cell volume, and greatest perturbations in genomic imprinting and expression of metabolic markers, while slower developing embryos displayed lower cell numbers, smaller cell volumes, but were best able to maintain genomic imprinting and showed expression levels of metabolic markers more similar to in vivo-derived embryos.

Conclusion: Our data support the quiet embryo hypothesis; embryos that maintain low levels of metabolic activity are best able to adapt to the suboptimal culture environment, resulting in the "healthiest" offspring.

\section{2-22 Every Body is a Story: Introducing Narrative Medicine for Canadian Contexts}

\section{Véronique Dorais Ram}

$\mathrm{MD} / \mathrm{PhD}$ Candidate, University of Calgary, BC, CAN

Background/Purpose: Current international literature demonstrates that physicians and other health care professionals with exposure to the humanities improve their critical thinking skills and bring enhanced sensitivity and analysis to their diagnostic reasoning. Medical programs, particularly in the United States, are incorporating "narrative medicine" into their curriculum to develop physicians with enhanced communication skills, stronger knowledge translation aptitudes, more astute and creative thinking tools, and lastly, the ability to alter public perception of physician practices. Existing studies illustrate that this thriving area of research can highlight the importance of biomedical narrative forms and promote the balance of treatment options between belief systems, patient history, and the technological possibilities offered by medical science. What are 
Canadian programs, however, doing to keep up with this trend?

Which studies clarify the principles of "narrative medicine" and how can we expand on the benefits, beyond better patient care, that this field of research holds for clinical practice? To explore these two primary questions, a summary of current programming and peer reviewed research is presented, along with suggestions for future research initiatives.

Methods: A review of the most updated (as of June 2012) Canadian and American medical program curriculums, and a literature search (Medline, UptoDate, MLA Bibliography, ERIC) was performed.

Results: The programming survey shows a substantial support for narrative medicine in the United States, and a growing interest at Canadian institutions (Alberta, Toronto, Dalhousie, and McGill). There is a wealth of research on the defining characteristics of narrative medicine and the benefits of integrating the study of narratives into medical schools; however, there is a lack of research on physician "stories" (for example, the clinical chart) as narrative medicine.

Conclusion: The current trend of narrative medicine offers a theoretically complex and fascinating new avenue to examine physician practices and medical ethics. More clinically focused projects, however, are needed to research the benefits of narrative medicine beyond medical education and physician health to consider clinical charts and physician documents as narratives themselves. Only through the analysis and data collection of both sides of the story can narrative medicine actually prove beneficial to the patientphysician relationship.

\section{2-23 Using ultrasonound to diagnose chronic exertional compartment syndrome}

\section{Sathish Rajasekaran ${ }^{1}$; Cole Beavis ${ }^{2}$; Abdel-Rahman Aly'; Dave Leswick ${ }^{3}$; Nadine Kanigan ${ }^{3}$}

${ }^{1}$ Department of PM\&R, University of Saskatchewan, ${ }^{2}$ Division of Orthopedic Surgery, Department of Surgery, University of Saskatchewan, ${ }^{3}$ Department of Medical Imaging, University of Saskatchewan, SK, CAN

Background/Purpose: Chronic exertional compartment syndrome (CECS) is commonly diagnosed with intracompartmental pressure testing. Several diagnostic imaging modalities have been described in the literature, but none are commonly used clinically. Using ultrasound to diagnose CECS has not been extensively studied in the literature. Our study aimed to test the hypotheses that CECS patients have an increased anterior compartment thickness compared to controls postexertion using ultrasound.

Methods: Four CECS patients (three males and one female) and 9 controls participated in the study. Our ultrasonography technique was first validated with a precision phase (10 controls) prior to scanning study subjects. CECS patients ran on a treadmill until symptomatic ( $\geq 5$ minutes) using a standardized protocol. Anterior compartment thickness (ultrasonography), and anterior compartment pressures were measured prior to exercise and at scheduled intervals afterwards. Controls underwent the same protocol without compartment pressure testing.

Results: Mean anterior compartment thickness in CECS patients vs. controls at rest was $2.63 \mathrm{~cm}$; $95 \%$ confidence interval (CI) $[2.11,3.14]$ vs. $2.18 \mathrm{~cm} ; 95 \% \mathrm{CI}[2.06,2.30], \mathrm{p}=.05$, at $0.5 \mathrm{~min}$ it was $3.17 \mathrm{~cm}$; $95 \% \mathrm{CI}[2.73,3.61]$ vs. 2.32 ; $95 \% \mathrm{CI}$ $[2.13,2.50], \mathrm{p}=.003$, at $2.5 \mathrm{~min}$ it was $3.17 \mathrm{~cm}$; $95 \%$ CI [2.73, $3.61]$ vs. $2.67 \mathrm{~cm} ; 95 \% \mathrm{CI}[2.10,2.43], \mathrm{p}=.003$, at $4.5 \mathrm{~min}$ it was 3.26; 95\% CI $[2.82,3.69]$ vs. $2.29 \mathrm{~cm}$; $95 \%$ CI [2.12, $2.45], \mathrm{p}=.003$, respectively. Mean percentage changes in anterior compartment thickness from rest in CECS vs. controls at $0.5 \mathrm{~min}$ was $21.3 \%$; $95 \% \mathrm{CI}[6.92,35.6]$ vs. $6.32 \%$; $95 \% \mathrm{CI}$ $[.094,12.5], \mathrm{p}=.011$, at $2.5 \mathrm{~min}$ it was $24.6 \%$; $95 \%$ CI [10.7, $38.5]$ vs. $4.22 \%$; $95 \% \mathrm{CI}[-1.85,10.3], \mathrm{p}=.003$, at $4.5 \mathrm{~min}$ it was $24.9 \%$; $95 \%$ CI $[14.3,35.5]$ vs. $5.08 \%$; $95 \%$ CI $[.813$, $11.0], \mathrm{p}=.003$, respectively. Compartment pressures were diagnostic of CECS using the modified Pedowitz criteria in all four CECS patients.

Conclusions: Ultrasonography reveals a significant increase in anterior compartment thickness in CECS patients compared to controls. Our study warrants further investigation into the potential role of using ultrasound to diagnose CECS clinically. 
2-24 Health care costs in people with diabetes and their association with glycemic control and kidney function

KA McBrien MD MPH ${ }^{1}$; BJ Manns MD MSc 1,2,3; B Chui $M D M S c^{4}$; SW Klarenbach MD MSct; D Rabi MD MScl,2,3; P Ravani MD PhD ${ }^{1,3}$; B Hemmelgarn MD PhD ${ }^{1,2,3}$; N Wiebe $\mathrm{MSc}^{4}$; Flora Au MSc ; F Clement PhD ${ }^{1,2,3}$

${ }^{1}$ Department of Medicine, ${ }^{2}$ Department of Community Health Sciences, ${ }^{3}$ Institute for Public Health, University of Calgary, AB, CAN

${ }^{4}$ Department of Medicine, University of Alberta, AB, CAN

Background: Suboptimal glycemic control, measured using hemoglobin Alc; proteinuria, measured using urinalysis; and reduced kidney function, measured using the estimated glomerular filtration rate, are independent predictors of adverse clinical outcomes, including cardiovascular morbidity and mortality, in people with diabetes. While the cost of diabetes is known to be higher for patients with comorbid illness, the link between cost and the laboratory measures noted above has not been firmly established or quantified.

Methods: We estimated the cumulative five-year cost of caring for people with diabetes using a province-wide cohort of adults with diabetes as of May 1, 2004. Costs included physician visits, hospitalizations, ambulatory care (emergency room visits, day surgery, and day medicine) and drug costs for people over 65 years of age. Using linked laboratory and administrative clinical and costing data, we determined the association between baseline glycemic control (hemoglobin A1c), proteinuria, and kidney function (eGFR), and five-year costs, controlling for age, socioeconomic status, duration of diabetes, and comorbidity.

Results: We identified 138,662 adults with diabetes. The mean five-year cost of diabetes across all members of the cohort was $\$ 26,978$ per patient excluding drug costs. The mean five-year cost for the subset of people over 65 , including drug costs, was $\$ 44,511$. Cost increased with worsening kidney function, presence of proteinuria, and suboptimal glycemic control (HbAlc $>7.9 \%$ ). Increasing age, Aboriginal status, socioeconomic status, duration of diabetes, and comorbidity were also associated with increasing cost.

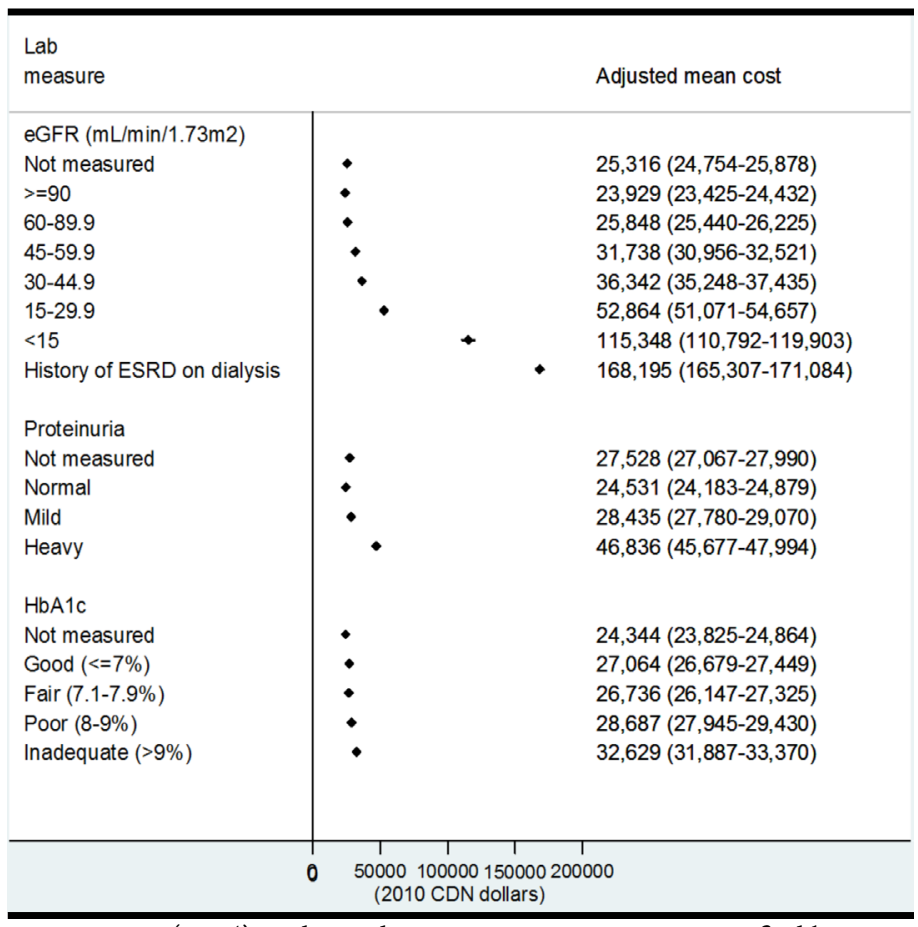

FIGURE 1 (2-24): Adjusted mean cost per patient, stratified by laboratory measure of relevance to patients with diabetes

Conclusions: The cost of caring for people with diabetes is substantial and is associated with suboptimal glycemic control, abnormal kidney function and proteinuria. Future studies should assess if improvements in the management of diabetes, assessed with laboratory-derived measurements, result in cost reductions.

\section{2-25 Epigenetic profiles in the sperm of male diabetic mice}

Michael Ke Wang BHSc; Paulina Rzeczkowska MSc; Mark R. Palmert MD PhD

Department of Genetics \& Genome Biology, The Hospital for Sick Childre

Background: Wildtype offspring of male mice with diabetes (Akita mice, C57BL/6-Ins2Akita/J) and wildtype C57BL/6 mothers develop metabolic and skeletal abnormalities in adulthood through non-genetic transmission.

Hypothesis: Transgenerational inheritance of phenotypic abnormalities from diabetic fathers to wildtype offspring occurs via transmission of abnormal epigenetic profiles (DNA methylation) in sperm of Akita mice. 
Methods: Sperm from the epididymides of both Akita and wildtype C57BL/6 males were collected in adulthood at 11 weeks of age. Purified DNA samples taken from sperm (Akita $\mathrm{n}=6$; wildtype $\mathrm{n}=6$ ) were analyzed for methylation changes at CpG islands using MALDI-TOF mass spectrometry on the Sequenom EpiTyper platform. Seven genes of interest, including Cdkn2a, Kcnq1, Slc30a3, Ppara, Pdx1, H19, and Fto, were analyzed. Because it is an imprinted gene, $\mathrm{H} 19$ was analyzed in sperm and epithelial tissue to ensure that the purification procedure was successful.

Results: T-tests with Bonferroni correction, comparing Akita and wildtype males, were conducted on the \% of methylated DNA at each CpG island. Significant differences at CpG islands on multiple genes (Slc30a3, Ppara, Pdx, and Kcnq1) were found. These differences ranged between $2 \%$ and $5 \%$. At all statistically significant CpGs, the CpGs of Akita males were hypomethylated compared to wildtype males.

Conclusion: Our results show that different DNA methylation patterns are found in sperm of diabetic mice compared to wildtype and suggest that this may be a mechanism that allows for transgenerational inheritance of phenotypes seen in the offspring of diabetic males.

1

2-26 Engineering interactions between the immune system and cardiac tissue during the early stages of myocardial infarction

\section{Jieun Kim ${ }^{1,2}$, Peter Zandstra ${ }^{2}$}

${ }^{1}$ Faculty of Medicine, ${ }^{2}$ Institute of Biomaterials and Biomedical Engineering, University of Toronto, Toronto, Canada

Background: Myocardial infarction (MI) is a leading cause of congestive heart failure[1]. MI induced inflammatory responses play a critical role in the formation of fibrotic scar in the myocardium, however, very little is known about the key cellular and molecular mechanisms in this process. In vivo MI model has been developed by surgical occlusion of left anterior descending coronary artery [2.3]. However, the invasive nature of surgical procedure can perturb the molecular basis of acute MI, and it is difficult to track physiological changes upon MI induction. Engineered heart tissue derived from human pluripotent stem cells (hPSCs) has become an ideal tool to study cardiovascular system in vitro [4-6]. Utilizing hPSC-derived cardiomyocytes (hPSC-CMs), an in vitro inter-tissue MI model can be developed. In particular, the transition between pro-inflammatory and anti-inflammatory stages can be studied in hypoxic conditions mimicking ischemia. Recent studies have shown that there are ranges of macrophages, involved in different stages of immune response. Classically activated macrophages (M1) are associated with inflammation while alternatively activated macrophages (M2) are involved in fibrosis formation and wound healing[7,8]. With their different roles, the relationship between MI progression and the transition from M1 to M2 phenotypes remain elusive. This study will focus on the interaction between hPSC-CMs and macrophage polarization, and could lead to the development of early stage pharmaceutical interventions capable of modulating heart disease progression.

Methods: To generate hPSC-CMs, two differentiation protocols were used: HES2 suspension culture with growth factors, and $\mathrm{H} 9$ and iPSC monolayer cultures with small molecules. Incubator with $5 \%$ oxygen was used to induce hypoxia. Monocytes were isolated from human cord blood, and macrophage colonizing stimulating factor and interleukins were added to differentiate and polarize macrophages.

Results: Levels of cardiac troponin T in hPSC-CM cultures were measured by flow cytometry and immunoflourescence. Preliminary qRT-PCR suggests hypoxia was induced in hPSC$\mathrm{CM}$ cultures. Cytokine and chemokine secretion profiles confirmed the macrophage polarization.

Conclusion: The preliminary data suggest the feasibility of generating of hPSC-CMs and polarized macrophages in vitro, and inducing hypoxia in hPSC-CMs. Various hypoxia models incorporating macrophages will be tested to mimic in vivo MI progression.

\section{References:}

1. Anesth Analg. 2002;95(6): 1788-92.Lloyd-Jones, D. M. et al. Lifetime risk for developing congestive heart failure: the Framingham Heart Study. Circulation 106, 3068-3072 (2002).

2. Pfeffer, M. A. et al. Myocardial infarct size and ventricular function in rats. Circulation Research 44, 503-512 (1979).

3. Degabriele, N. M. Critical appraisal of the mouse model of myocardial infarction. Experimental Physiology 89, 497-505 (2004).

4. Iyer, R. K., Chiu, L. L., Reis, L. A. \& Radisic, M. Engineered cardiac tissues. Current Opinion in Biotechnology 22, 706-714 (2011).

5. Hansen, A. et al. Development of a drug screening platform based on engineered heart tissue. Circulation Research 107, 35-44 (2010). 
6. Schaaf, S. et al. Human Engineered Heart Tissue as a Versatile Tool in Basic Research and Preclinical Toxicology. PLoS ONE 6, e26397 (2011).

7. Gordon, S. Alternative activation of macrophages. Nat. Rev. Immunol. 3, 23-35 (2003).

8. Gordon, S. \& Martinez, F. O. Alternative activation of macrophages: mechanism and functions. Immunity 32, 593-604 (2010).

\section{2-27 The public health consequence of smear-positive pulmonary tuberculosis in patients with typical and atypical chest radiographs}

\section{Lau $A^{1,2}$; Barrie J' Winter $C^{1}$; Kunimoto $D^{1}$; Elamy $H^{1}$; Tyr - rell $G^{1}$; Long $R^{1}$}

${ }^{1}$ Tuberculosis Program Evaluation and Research Unit, Department of Medicine, Department of Radiology, Department of Public Health Sciences, University of Alberta and ${ }^{2}$ Leaders in Medicine Programme, University of Calgary Faculty of Medicine

Background: Using currently available digital technology, it should be possible to automate the detection of 'typical' postprimary tuberculosis (TB) on chest radiograph (CXR). Development of such a detection system is warranted if it can be demonstrated that patients with 'typical' (vs 'atypical') CXRs are responsible for most public health consequences (recently infected persons amongst close contacts; secondary cases amongst all cases).

Methods: Over 30 months beginning January 1, 2006, all adults (age $>14$ years) diagnosed with smear-positive pulmonary TB in Alberta, were identified in the TB Registry. Patient demographics and mycobacteriology were abstracted from public health records and the Provincial Laboratory for Public Health. Pre-treatment posterior-anterior and lateral CXRs were assembled and scored by 3 independent readers as 'typical' (having an upper lung zone infiltrate, with or without cavitation, but no discernable intrathoracic adenopathy) or 'atypical' (all others). The public health consequences, confirmed using molecular diagnostics, from each group were compared.

Results: There were 88 adults with smear-positive pulmonary TB, of whom $62(70.5 \%)$ had a 'typical' CXR. Patients with 'typical' CXRs had, on average, larger bacillary burdens and metabolically more active bacteria (larger semi-quantitative smears; shorter times-to-culture-positivity) than patients with 'atypical' CXRs. More importantly, they were responsible for most public health consequences: 0.39 vs 0.04 child-aged tu- berculin skin test (TST) converter per source case, $p=0.008$; 0.83 vs 0 child-aged TST converter per Canadian-born source case, $\mathrm{p}=0.009$; and 35 vs 4 secondary cases.

Conclusion: Adult smear-positive pulmonary TB patients with 'typical' CXRs are responsible for most public health consequences. Accordingly, the development of an automated radiographic TB detection system is warranted.

\section{2-28 Influenza infects lung microvascular endothelium leading to microvascular leak}

Susan M. Armstrong ${ }^{1}$; Changsen Wang ${ }^{2}$; Jayesh Tigdi'; Xiaoe Si3; Carlo Dumpit'; Steffany Charles ${ }^{2}$; Asela Gamagel; Theo J. Moraes $^{1,3}$; Warren L. Lee L $^{1,2,4}$

${ }^{1}$ Institute of Medical Science, University of Toronto; ${ }^{2}$ Keenan Research Centre of the Li Ka Shing Knowledge Institute of St. Michael's Hospital (Toronto); ${ }^{3}$ Hospital for Sick Children, Toronto; the ${ }^{4}$ Division of Respirology and Interdepartmental Division of Critical Care Medicine, Faculty of Medicine, University of Toronto

Background/Purpose: Severe influenza infections are complicated by acute lung injury, a syndrome of pulmonary microvascular leak. The pathogenesis of this complication is unclear. We hypothesized that human influenza could directly infect the lung microvascular endothelium, leading to loss of endothelial barrier function.

Methods: We infected human lung microvascular endothelium with both clinical and laboratory strains of human influenza. Permeability of endothelial monolayers was assessed by spectrofluorimetry and by measurement of the transendothelial electrical resistance. We determined the molecular mechanisms of flu-induced endothelial permeability and developed a mouse model of severe influenza.

Results: We found that both clinical and laboratory strains of human influenza can infect and replicate in human pulmonary microvascular endothelium, leading to a marked increase in permeability. This was caused by apoptosis of the lung endothelium, since inhibition of caspases greatly attenuated influenzainduced endothelial leak. Remarkably, replication-deficient virus also caused a significant degree of endothelial permeability, despite displaying no cytotoxic effects to the endothelium. Instead, replication-deficient virus induced degradation of the tight junction protein claudin-5; the adherens junction protein VE-cadherin and the actin cytoskeleton were unaffected. Over- 
expression of claudin-5 was sufficient to prevent replicationdeficient virus-induced permeability. The barrier-protective agent formoterol was able to markedly attenuate flu-induced leak in association with dose-dependent induction of claudin5. Finally, mice infected with human influenza developed pulmonary edema that was abrogated by parenteral treatment with formoterol.

Conclusion: We describe two distinct mechanisms by which human influenza can induce pulmonary microvascular leak. Our findings have implications for the pathogenesis and treatment of acute lung injury from severe influenza.

\section{2-29 The Stretpcoccus Antibiotic Resistome of Adult Cystic Fibrosis Patients Results from Mutation and Horizontal Gene Transfer}

Christina S. Thorntonac, Margot E. Grinwisa, Christopher D. Sibleyac, Harvey R. Rabin ${ }^{a b}$ and Michael G. Surettead

aDepartment of Microbiology, Immunology and Infectious Diseases, bAdult Cystic Fibrosis Clinic, cLeaders in Medicine Program, Faculty of Medicine, University of Calgary, Calgary, Alberta, Canada. dDepartments of Medicine and Biochemistry and Biomedical Sciences, McMaster University, Hamilton, Ontario, Canada

Rationale: Cystic fibrosis (CF) is the most common lethal genetic disease among Caucasians. 90\% of CF patients succumb to pulmonary failure from chronic respiratory infections. Traditionally, research has focused on a narrow spectrum of microorganisms thought to be principal pathogens such as Pseudomonas aeruginosa. However, emerging species, such as the Streptococcus genus, have been shown to be overlooked pathogens. Within the streptococci, there is the potential for an antibiotic resistome due to constant pressure from several prescribed antibiotics as well as the naturally competent nature of the bacteria. Earlier studies done on the microbiome within $\mathrm{CF}$ has shown the presence of multiple isolates from several novel species of streptococci, which may play a role in CF exacerbation. As such, the objectives were to determine rates of resistance among common antibiotics in $\mathrm{CF}$ and to elucidate molecular mechanisms of resistance.

Methods: In this study, 459 streptococcal isolates from 68 adult CF patients comprising of 16 novel and typed species underwent susceptibility testing for nine antibiotics. Molecular mechanisms of resistance for the macrolides were determined by a PCR-based screen or DNA sequencing.
Results: Resistance rates were the greatest for macrolide antibiotics at $51.6 \%$ for erythromycin and $56.4 \%$ for azithromycin, but with novel isolates closer to $80 \%$. As such, macrolide resistance was looked at in closer detail. The two most common mechanisms of macrolide resistance within the streptococci acquired by horizontal gene transfer, the mef (efflux pump) and erm (target site methylation) accounted for only $53 \%$ of resistant isolates. Interestingly, the rarely described mechanism of $23 \mathrm{~S}$ ribosomal point mutations, the target site of macrolides, accounted for $47 \%$ of resistant isolates.

Conclusions: The prevalence, species distribution and influence of therapy on resistance profiles suggest complex ecological interactions among the streptococci in the airways of CF patients with mutation, rather than solely horizontal gene transfer, being a significant mechanism of acquired antibiotic resistance in some species within this community. This is in contrast to similar studies done within CF demonstrating resistance solely by acquisition of virulence determinants. This study also illuminates the potential roles for the Streptococcus genus in CF disease progression.

\section{2-30 Effect of PGC-1alpha on alpha-synuclein induced toxicity in mice}

\section{Katherine Y. Li', Carine Ciron'; Bernard Schneider'; Patrick Aebischer ${ }^{2}$}

${ }^{I}$ Faculty of Medicine, University of Ottawa, Ottawa, ON, CAN

2Brain Mind Institute, Ecole Polytechnique Fédérale de Lausanne, Lausanne, Switzerland

Background/Purpose: Parkinson's disease (PD) is a neurodegenerative disorder characterized by the progressive loss of dopaminergic neurons in the substantia nigra pars compacta (SNpc). Accumulation of alpha-synuclein and its familial mutants sensitize dopaminergic neurons to oxidative stress and damage induced by mitochondrial toxins. Therefore, pathways controlling mitochondrial function rapidly emerge as potential therapeutic targets. In this context, peroxisome proliferatoractivated receptor gamma coactivator-1 alpha (PGC-1 $1 \alpha$ ), a master regulator of mitochondrial biogenesis and metabolism, appears as a possible major target. Here, we investigate whether or not the local expression of PGC- $1 \alpha$ in the SNpc of PGC-1 $\alpha$ knock-out mice (PGC1 KO) can prevent $\alpha$ Syn-induced toxicity by restoring normal PGC- $1 \alpha$ levels. 
Methods: PGC1 KO mice were co-injected in the right SNpc with AAV2/ 6 vectors coding for PGC- $1 \alpha$ and wild-type human alpha-synuclein $(\alpha$ SynWT). A non-coding vector (NCV) and $\alpha$ SynWT co-injection was served as control. Animals were sacrificed 6 months post-vector injection. $4.25 \mu \mathrm{m}$-thick coronal sections were harvested. Slices were processed for immunohistological treatment.

Results: Local expression of PGC1 in nigral dopaminergic neurons of PGC1 KO mice prevents the neuronal loss induced by $\alpha$ SynWT overexpression.

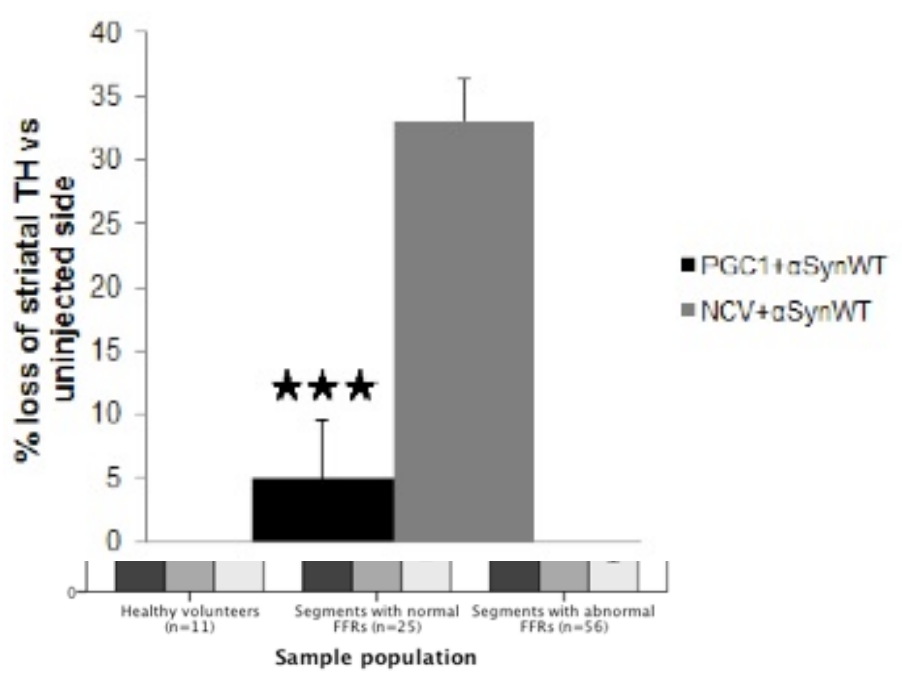

FIGURE 1 (2-30): Decreased loss of striatal TH-positive neurons in response to the local expression of PGC1 6-months post-vector injection. Error bars represent SEM of the sample. t-test:

PGC1+aSynWT n=8, NCV+ + SynWT $n=10 . \star \star \star$ p $<0.01$

Conclusion: Local expression of PGC1 in nigral dopaminergic neurons of PGC1 KO mice prevents the neuronal loss induced by $\alpha$ SynWT overexpression. This finding, with further evaluations, holds promising therapeutic potentials for PD treatment.

\section{2-31 Prevalence Of Self-Reported Injury Prone Behav- iours In Staff Physicians}

\author{
Manveer Khanijoun B.Eng'; Raviraj Singh BHSc ${ }^{2}$; Navneet \\ Singh, MD'; Joanne Banfield RN B $A^{4}$ \\ ${ }^{1}$ Faculty of Engineering, University of Alabama at Birming- \\ ham, Birmingham, AL,USA \\ ${ }^{2}$ Faculty of Sciences, University of Western Ontario, London, \\ ON, CAN \\ ${ }^{3}$ Faculty of Medicine, University of Toronto, Toronto, ON, \\ CAN \\ ${ }^{4} \mathrm{RBC}$ First Office for Injury Prevention
}

Purpose: Injury-prone behaviours are highly associated with traumatic brain injury (TBI) and spinal cord injury (SCI). TBI and SCI result in mortality, disability, health care costs and comorbidities. Injury-prone behaviours in physicians are understudied. Thus, we aim to investigate the prevalence of injuryprone behaviors in staff physicians at a single academic tertiary care centre.

Methods: A questionnaire was administered prospectively between February and March 2008 to staff physicians via official e-mail at a single academic tertiary care centre with an annual census of 29,254. The questionnaire utilized a total of 17 items from the Centre for Disease Control's Behaviour Risk Factor Surveillance System and Colorado State's Surveillance Survey, both of which are valid and reliable tools in current use. Categorical variable items employed 5-point Likert or dichotomous (i.e., yes or no) response options. Chiefly descriptive analysis was used. The chi-squared and ANOVA tests were used to compare categorical and continuous variables, respectively. Where applicable, $\mathrm{p}$-values were included.

Results: Staff physicians reported speeding sometimes (65\%, $\mathrm{n}=68 / 105)$, drinking too much and driving in the past month $(3 \%, n=3 / 105)$, being a passenger in a vehicle with a driver that has had too much to drink in the past month $(4 \%, n=4 / 105)$, never wearing bike helmets $(6 \%, n=6 / 105)$, almost never crossing at an intersection $(19 \%, \mathrm{n}=20 / 105)$, almost never wearing a life jacket while boating $(10 \%, \mathrm{n}=11 / 105)$, and not having $\mathrm{CO}$ detector in the home $(17 \%, \mathrm{n}=18 / 105)$ as shown in Table 1. Respondents were male $(58 \%, n=61 / 105)$, married $(83 \%$, $\mathrm{n}=87 / 105)$, and between 30 to 60 years of age $(90 \%, \mathrm{n}=95$ /105).

Conclusions: Staff physicians overall demonstrated a low selfreported prevalence of injury-prone behaviors. Larger studies that address the limitations inherent in this study are necessary 
to further explore and clarify self-reported injury-prone behaviours.

\section{2-32 Nlrp3 promotes cardiac fibrosis and myofibroblast differentiation independently from the Inflammasome}

\section{Nathan A Bracey; Paul L Beck; Daniel A Muruve; Henry J \\ Duff}

Libin Cardiovascular Institute, Faculty of Medicine University of Calgary, Calgary Alberta, Canada

Background: Hypertension-induced myocardial fibrosis is a significant cause of heart failure. This fibrotic signaling occurs through the differentiation of resident fibroblasts into contractile myofibroblasts. These wound-healing cells are found only in the diseased heart, and secrete pro-fibrotic substances that increase cardiac stiffness. While the longstanding consequences of cardiac fibrosis are well established, the cellular and molecular mechanisms behind myofibroblast recruitment remain unknown.

Nlrp3 is a pattern recognition receptor that is activated by danger signals. In immune cells, Nlrp3 signals for the assembly of the inflammasome, a multi-protein complex that leads to the secretion of pro-inflammatory cytokines. While many studies have looked at intra-cardiac activation of the inflammasome, the role of Nlrp3 in structural heart cells has been unexplored. We hypothesized that Nlrp3 within fibroblasts participates in the differentiation of myofibroblasts to promote cardiac fibrosis.

Methods and Results: Differentiation of primary murine cardiac fibroblasts into myofibroblasts in vitro with angiotensin II (angII) or TGF $\beta$ resulted in a SMAD dependent increase in Nlrp3 protein. This increase occurred without activation of the inflammasome, evidenced by the absence of IL- $1 \beta$ secretion or caspase-1 processing. Nlrp3 deficient cardiac fibroblasts exhibited reduced $\alpha$-smooth muscle actin ( $\alpha \mathrm{SMA})$ compared to wild-type (WT) cells, indicating impaired myofibroblast formation. This was paralleled by reduced activation of profibrotic SMAD signaling, where Nlrp3-/- fibroblasts showed significantly reduced SMAD2/3 phosphorylation following TGF 3 . Despite altered SMAD signaling, Nlrp3-/- cells exhibited preserved ERK1/2 and AKT signaling, indicating a SMAD-specific role for Nlrp3. In vivo, chronic angII infusion in WT mice for 28 days resulted in elevated blood pressure and patchy interstitial myocardial fibrosis on histology. Nlrp3-/mice developed hypertension, but with fewer fibrotic regions and reduced induction of $\alpha$ SMA.
Conclusion: These results demonstrate a critical role for Nlrp3 in myofibroblast differentiation within the heart. They implicate a novel role for Nlrp3, independently from the inflammasome in the pathogenesis of fibrotic heart disease. Thus, Nlrp3 may represent a novel therapeutic target for the treatment of cardiac fibrosis and its resulting heart failure.

\section{2-33 The Impact of Restricting Ranibizumab Coverage in Ontario, Canada for Macular Edema from Vein Oc- clusions and Diabetic Retinopathy}

Christina Leung MD, FRCSC' ; Yao Wang BHSc ${ }^{2}$; Mario Ventresca MD, FRCSC ${ }^{3}$; Michael Brent MD, FRCSC; Robert Devenyi MD, MBA, FRCSC', Wai-Ching Lam MD, FRCSCI ${ }^{1}$ Department of Ophthalmology and ${ }^{2}$ Medicine at the University of Toronto, and ${ }^{3}$ Port Colborne, Ontario

Background/Purpose: For 3 years, the Ontario government covered ranibizumab costs for all patients over the age of 65 years regardless of the indication. However, on May 19th, 2011, the government ceased coverage of ranibizumab for centre-involving diabetic macular edema (C-DME) and macular edema following retinal vein occlusions (ME-RVO), requiring patients to find alternative funding for ranibizumab or switch to another treatment. The objective of this study is to analyze the impact of this policy change.

Methods: A retrospective chart review of 4 vitreoretinal practices identified patients who received at least 3 intravitreal ranibizumab (IVR) injections from February 19 to May 19, 2011 for either C-DME or ME-RVO and had a minimum 6 months follow-up thereafter. The patients' demographics, diagnosis, treatments, best-corrected visual acuity (BCVA), and central foveal thickness (CFT) were recorded up to 6 months after May 19th. The primary outcome was a qualitative observation of the distribution of treatments chosen as second-line therapy. Secondary outcomes included differences in the mean change in BCVA and CFT 6 months after implementation of the policy in eyes that continued on IVR versus eyes that switched to another therapy. The frequency of clinic visits and adverse events were also observed between the two groups.

Results: Fifty-six eyes met the study criteria: 31 eyes had CDME and 25 eyes had ME-RVO. Of the 56 eyes, 15 continued on IVR, 25 received intravitreal bevacizumab (IVB), 3 received a combination of IVB and an Ozurdex implant, 2 received in- 
travitreal triamcinolone (IVT), 2 received a combination of IVR and macular laser photocoagulation (MPC), 3 received a combination of IVB and MPC, 1 received a combination of IVT and MPC and 5 received MPC alone.

After 6 months, eyes with C-DME that continued with IVR had a visual improvement of 2.2 lines from baseline compared to 0.4 lines in eyes that switched treatments $(\mathrm{P}=0.02)$. A difference was also seen in the mean CFT change from baseline between these two groups: $-201.3 \mu \mathrm{m}$ in the group that continued IVR versus $-16.2 \mu \mathrm{m}$ in the group that switched treatments $(\mathrm{P}=0.01)$. Eyes with ME-RVO that continued on IVR showed no difference in visual improvement $(\mathrm{P}=0.93)$ or reduction of CRT $(\mathrm{P}=0.95)$ compared to those that switched treatments. There was no significant difference in the number of clinic visits or adverse events between the two groups.

Conclusions: Discontinued government funding of ranibizumab for C-DME and ME-RVO resulted in considerable variability in the therapeutic management of these diseases. Although most patients continued on some type of anti-vascular endothelial growth factor agent, our findings suggest that those with C-DME who continued with IVR had better outcomes. This effect, however, was not seen in eyes with ME-RVO. Further investigation to validate this trend and determine the reasons for such findings is warranted.

\section{2-34 Whole organ MRI score (WORM) of the hip: de- velopment of a diagnostic tool for predicting surgical outcome}

\section{Anthony Vo, Kyle Kemp, Paul Beaulé, Nathan Sacevich, Kawan Rakbra, Mark Schweitzer}

University of Ottawa

Purpose: Hip arthroscopy has now evolved into a common orthopedic procedure for the treatment of labral pathology. However clinical results can vary with state of the articular cartilage on plain radiographs being solely used to predict clinical outcome. A more complete multi-planar assessment of the hip joint (WORM) including bone, synovium may provide a more sensitive diagnostic tool to assist appropriate selection of patients for hip arthroscopy. The purpose of this study was to develop a WORM score for the hip and determine its' potential usefulness in predicting the clinical outcome of patients undergoing hip arthroscopy.

Methods: Forty patients (10 males, 30 females, mean age $=38.6 \pm 13.2$ years, range $=17$ to 63 ) were recruited with hip pain and a diagnosed labral tear on MRI. MR images were acquired on a $3 \mathrm{~T}$ MR scanner according to a protocol designed for hip pathology. Each hip was divided into three areas on the acetabular side and four on the femoral side. Hip joints were graded by a musculoskeletal radiologist; providing a preoperative WORM score, with a high score indicating increased disease. Correlations for individual structures, each area, and total WORM scores were calculated for baseline and follow-up (1 year) WOMAC.

Results: Greatest WORM scores were seen in the superolateral acetabular $(56.2 \pm 13.8)$ and supero-anterior femoral head (38.4 \pm 12.1$)$ zones. Mean WOMAC function improved significantly from $64.2 \pm 21.1$ (pre-op) to $77.5 \pm 19.9$ (1 year) $(\mathrm{p}=0.04)$. At follow-up, the total WORM score was significantly correlated with stiffness $(r=-0.44, p=0.03)$ function $(\mathrm{r}=-0.41, \mathrm{p}=0.04)$ on the WOMAC and correlated with age $(\mathrm{r}=0.42, \mathrm{p}=0.02)$.

After item reduction ( 43 to 8 items), WORM score correlation with one year WOMAC improved, with significant values observed in all domains: pain $(\mathrm{r}=-0.48, \mathrm{p}=0.02)$, stiffness $(\mathrm{r}=-0.55, \mathrm{p}=0.006)$, function $(\mathrm{r}=-0.56, \mathrm{p}=0.005)$, total $(\mathrm{r}=-0.55, \mathrm{p}=0.006)$. Univariate analysis showed that the presence of CAM FAI at surgery $(\mathrm{p}=0.04)$ and a lower preoperative WORM score $(\mathrm{p}=0.006)$ were associated with greater WOMAC total follow-up scores. WOMAC and WORM scores were not correlated with gender, BMI, or Tonnis grade. When controlling for these factors (multivariate analysis), CAM FAI and WORM remained significant $(\mathrm{r}=0.42, \mathrm{p}=0.01$ and $\mathrm{r}=-0.55, \mathrm{p}=0.001$ respectively).

Conclusion: Our preliminary results indicate that the preoperative condition of various structures of the hip joint considered as whole can predict post-operative patient function at one-year. Item reduction led to further improvements in correlation, suggesting that certain items of the WORM score are not associated with subjective reports of post- operative function. 


\section{2-35 A Randomized Clinical Trial Comparing Breast and Abdominal Related Morbidity of DIEP and SIEA Flaps}

\section{Peters, Blair R}

University of Manitoba

Background: The deep inferior epigastric perforator flap (DIEP) is the current standard of care in breast reconstruction. The newer superficial inferior epigastric artery flap (SIEA) is felt to be an improvement as it does not damage the abdominal wall. The SIEA flap unfortunately is reported to have smaller vessels which put the flap at higher risk of developing flap loss and fat necrosis. The uncertainty regarding the tradeoffs inherent in the choice of procedure has not been resolved. As such we have aimed to perform a randomized single blinded trial to evaluate the abdominal and breast related morbidity associated with DIEP and SIEA flaps.

Methods: A blinded, randomized clinical trial is being performed involving Manitoban women over 18 years undergoing unilateral or bilateral breast reconstruction. Women are randomized to either receive the DIEP or SIEA flap procedure. Objective isokinetic abdominal muscle and back extensor strength testing is being done pre-operatively and 3, 6 and 12 months post-operatively. A validated abdominal wall and breast outcome questionnaire (Breast- $\mathrm{Q}$ ) is being administered pre-operatively and at 3 and 12 months post. Secondary outcomes measured include: fat necrosis, flap loss, abdominal wound breakdown, seroma rate and length of hospital stay. Statistical analyses include a combination of parametric and non-parametric tests.

Results: Preliminary analyses have shown some significant post-operative decreases in the DIEP abdominal assessments. Some of the complication rates appear to be higher in the SIEA group, however sample sizes are currently too small for the differences to be called significant. At this point there does not appear to be any significant difference in the intraoperative findings between groups. This is an important finding in itself. These preliminary results challenge the assumption that the superficial inferior epigastric vessels (when present) are too small to support a tissue flap, and suggest that more patients may be candidates for the SIEA flap procedure than currently thought.

Conclusions: Although the data obtained is preliminary we believe that upon completion of this study, we will go further than others in controlling variability and providing pure data for analysis. By examining outcomes on both donor site morbidity and breast flap complications, we hope to clearly delineate the benefits and tradeoffs inherent in the choice of procedure.

\section{2-36 Matching motoneuron and muscle fiber pheno- type: the chicken or the egg?}

Magown Philippe JS1,2; Rafuse VF2; Brownstone RM1,2

${ }^{1}$ Department of Surgery (Neurosurgery), Dalhousie University, Halifax, CAN

${ }^{2}$ Anatomy \& Neurobiology, Dalhousie University, Halifax, CAN

Alpha motoneurons can be classified as fast or slow based on their electrophysiological properties. Motoneurons and their muscle unit phenotypes match. However, it is unclear if motor unit phenotype is determined by the motoneuron or the muscle fiber. Developmental studies on avian and rodent embryos suggest that muscle fiber phenotype is innervationindependent. On the other hand, cross-reinnervation studies indicate that motoneurons direct muscle fiber phenotype. In order to investigate this further, mouse immature motoneurons were co-cultured with either fast or slow myotube populations. We hypothesized that electrophysiological properties of recorded motoneurons would match the muscle fiber types with which they were cultured.

Embryonic stem cell-derived motoneurons were plated on either slow myotubes obtained from the external adductor muscle or fast myotubes harvested from the pectoral muscle of E10 chick embryos. After one month, muscle contractions were chemically blocked to study motoneuron properties using patch electrodes.

The two sets of motoneurons had similar whole cell capacitances, input resistances, and rheobases. Medium afterhyperpolarization amplitude, half-width, and half-decay time were also similar in both conditions. Firing frequencies were nearly identical with first interval maximal frequencies of 150 $\mathrm{Hz}$ and steady state firing frequencies of $30 \mathrm{~Hz}$.

We conclude that one month old embryonic stem cell derived motoneurons demonstrate similar electrophysiological properties whether grown on fast or slow myotubes. Such findings indicate that either motoneuron phenotype is independent of the phenotype of the innervated muscle fibers or that ES cell derived motoneurons are unable to change their phenotype in response to different muscles types. 


\section{2-37 Diffusion tensor imaging in multiple sclerosis: A method of differentiating symptomatic and asympto- matic cervical cord lesions}

\section{Soraya Mehdizadeh; Karunanithi Rajamanickam; Aparna Gupta; Roxana Cruce; Arturo Cardenas Blanco; Mark S.} Freedman; Santanu Chakraborty; Thanh Nguyen; Eve C. Tsai Faculty of Medicine, University of Ottawa and The Ottawa Hospital Research Institute

Background/Purpose: Diffusion tensor imaging (DTI) is a novel magnetic resonance imaging technique that can image specific fiber tracts in-vivo and has been used to examine the spinal cord in patients with multiple sclerosis (MS). Studies have shown that DTI can identify cervical cord damage in MS, however, correlation with clinical symptoms is limited. Our objective was to see if DTI could differentiate symptomatic from asymptomatic MS cervical cord lesions.

Methods: Fourteen MS patients with cervical cord lesions (10 female, 4 male; mean age: $46, S D=13.26)$ and eight healthy controls ( 6 female, 2 male; mean age: $30, S D=5.09$ ) were evaluated in this study. Sagittal T2-weighted images and DTI of the cervical cord were obtained using a 3T Siemens Magnetom Trio MR imager.

Within 24 hours of their imaging, all patients were neurologically assessed using the American Spinal Injury Association classification of spinal cord injury and the Expanded Disability Status Scale. Patients were classified as having symptomatic or asymptomatic cervical spinal cord lesions based on their clinical evaluation. DTI measures of mean radial diffusivity, (RD) and mean diffusivity (MD) were assessed at the MS lesion, peri-lesion and normal appearing white matter (NAWM) levels as identified by the $\mathrm{T} 2$ weighted images.

Results: Patients with symptomatic lesions had a significantly higher mean radial diffusivity $(\mathrm{RD})$ in lesion sites $(\mathrm{p}=0.047)$, as well as a higher mean diffusivity $(\mathrm{MD})(\mathrm{p}=0.041)$ and mean $\mathrm{RD}(\mathrm{p}=0.034)$ in peri-lesional sites compared to asymptomatic patients. No significant differences in DTI measures were found in NAWM between these two groups. See Figure 1 for tractography and corresponding T2-weighted images of the cervical cord in symptomatic and asymptomatic MS patients.

Conclusion: Our study demonstrated that DTI measures could differentiate symptomatic from asymptomatic spinal cord lesions in patients with multiple sclerosis. Further studies will evaluate the use of DTI in monitoring disease progression and disability in MS patients with cord lesions.

\section{2-38 Slit2/robo-1 signaling in monocyte recruitment and adhesion: a target in vascular inflammation.}

\section{Ilya Mukovozova; Y.W. Huanga, G.Y. Liua; S. Patela and L. Robinsona}

Cell Biology Program, The Hospital for Sick Children Research Institute, Toronto, ON, CAN

Background: Chronic inflammation is characterized by prolonged infiltration of mononuclear leukocytes, including monocytes and lymphocytes. Persistent vascular inflammation and the subsequent cellular influx is a hallmark in the pathogenesis of atherosclerosis. In both animal models and human subjects, inhibiting monocyte recruitment partially prevents atherosclerosis and its clinical manifestations. The Slit family of secreted proteins, together with their transmembrane receptor Robo, repel migrating neurons during CNS development. Recently, Slit2 was shown to inhibit the migration of other cell types, including vascular smooth muscle cells, breast cancer cells, and circulating human neutrophils.

Purpose: The effects of exogenous Slit2 on monocyte recruitment and adhesion under shear flow, along with the corresponding intracellular signaling pathways, will be investigated. We hypothesize that Slit2 will decrease monocyte adhesion and recruitment both in vitro and in vivo, by diminishing arrest on activated endothelium under shear flow. Furthermore, we hypothesize that Slit 2 acts by inhibiting activation of Rho GTPases Cdc42 and Rac1, required for the actin-driven stabilization of firm adhesions.

Methods: Monocyte migration in vivo was investigated using a murine model of irritant-induced peritonitis. Adhesion assays under shear flow were utilized to investigate the effect of Slit2 on monocyte capture, rolling and arrest. Adhesion was tested to both activated endothelial cells and immobilized immunoglobulin superfamily ligands, including ICAM-1 and VCAM1. Western blotting was used to determine the intracellular signaling events mediating the effects of Slit 2 on monocytes. Results: Slit2 administration decreased monocyte recruitment in vivo $(\mathrm{P}<0.001)$. In addition, Slit2 inhibited monocyte adhesion to TNF- $\alpha$ stimulated endothelial monolayers under static and shear flow conditions $(\mathrm{P}<0.01)$. Finally, Slit2 decreased the 
arrest of monocytes on activated endothelium $(p<0.05)$, although rolling and capture was not affected.

Conclusions: These experiments demonstrate that Slit2 inhibits monocyte recruitment in vivo. Slit 2 was also shown to inhibit monocyte adhesion to activated endothelial cells and immobilized ICAM-1/VCAM-1. This may allow the use of Slit2 as a novel anti-inflammatory therapy to locally target the leukocyte adhesion cascade and prevent chronic monocytes infiltration in atherosclerotic lesions.

\section{2-39 Waiting less for hip and knee replacement: Cana- dian orthopaedic surgeons' acceptability of single-entry models as a means of managing patients}

\section{Damani Z, Conner-Spady B, Noseworthy $T$}

Western Canada Waiting List Investigators, Department of Community Health Sciences, University of Calgary, 3330 Hospital Drive NW Calgary, AB T2N 4N1 Canada

Background/Purpose: Single-entry models (SEMs) have been shown to reduce waiting times, increase equity and general satisfaction by providing patients access to necessary services through a single point-of-entry. However, it is not known if SEMs are a valued or acceptable waiting time management strategy for patients and their clinicians.

The purpose is to understand surgeon acceptability of SEMs by Canadian surgeons (and under what conditions) receiving referrals for hip and knee replacement (H\&KR).

Methods: Experienced surgeons were chosen from a larger study, came from 3 Provinces across Canada, and 68\% accepted patients from a pooled list. Nineteen were interviewed regarding their views on SEMs as a means to access scheduled H\&KRs. Academic/community-based, as well as urban/ruralbased orthopaedic surgeons from Alberta, Manitoba and Nova Scotia were included. Data were collected through semistructured telephone interviews.

Results: Nineteen surgeons were interviewed. Eighty-four percent $(16 / 19)$ rated SEMs as acceptable ( $47 \%$ very/37\% somewhat). Rural and urban $(4 / 19,15 / 19)$ as well as community and academic $(8 / 19,11 / 19)$ surgeons were accepting of the basic tenets of SEMs, expressing an appreciation for the improved patient care, reduced waiting times afforded by SEMs. They disagreed and would oppose SEMs on the grounds of lack of continuity of care, surgeon autonomy/control, lack of pa- tient choice, inappropriate patient referrals and lack of funding.

While responses from rural and urban surgeons were similar, rural surgeons $(80 \%$ were general orthopaedic surgeons, with many non-H\&KR patients) seemed less informed about SEMs and more reluctant to implement them, citing lesser demand for H\&KR than in urban areas and less support/funding being available to them.

Conclusion: Though many surgeons appear to accept the value of SEMs and are in favour of implementing them, acceptability appears conditional, not uniform. There are particular critical success factors, which if considered, would increase surgeon acceptability and, accordingly, improve the opportunity for successful implementation, with generalizability to other scheduled services: considerations needed for the implementation of SEMs and important incentives in making SEMs acceptable to surgeons and their patients include improving the appropriateness of referrals, trained staff, funding, standardized continuum of care, central intake, reduced waiting times and more surgeon control.

Funding: University of Calgary Program for Undergraduate Research Experience (PURE) Award Western Canadian Waiting List Investigators CIHR Emerging Team Grant

\section{2-40 No obvious behavioural phenotype in initial screen of a Neuronal calcium sensor-1 knockout mouse- line}

Enoch $\mathrm{Ng}^{1}$; Joanna Hermainski²; Olaf Pongs ${ }^{2}$; Albert HC Wong'; John C Roder

${ }^{1}$ University of Toronto

${ }^{2}$ University of Hamburg

Background/Purpose: Neuronal Calcium Sensor-1 (NCS-1) is an N-terminally myristoylated EF-hand protein involved in neurotransmission, synaptic plasticity, neurite outgrowth, Gprotein coupled receptor signaling, ion channel regulation, learning and memory. Knocking out the $N c s-1$ analogue in $C$. elegans impairs associative memory, while overexpression enhances learning. Selectively overexpressing $N c s-1$ in mouse dentate gyrus enhances exploration and spatial learning and memory. Moreover NCS-1 has been implicated in disease such as schizophrenia, bipolar disorder and autism. To better understand the role of NCS-1 in whole animal behaviour, a Ncs-1 
knockout line was generated and screened for behaviours relevant to neuropsychiatric disease.

Methods: The Ncs-1 knockout line was generated by crossing a Cre-deleter line with a Ncs-1 knockin line that had critical exons of Ncs-1 flanked by loxP sites. 8-12 week old mice wildtype, heterozygous or null for $N c s-1$ underwent a battery of behavioural tests. The order of testing was: open field activity, light-dark box, Y-maze spontaneous alternation, pre-pulse inhibition, fear conditioning and the tail-suspension test.

Results: All genotypes performed similarly in the open field, suggesting no locomotor deficits or hyperactivity associated with knocking out $N c s-1$ in mice. No differences between genotypes in the light-dark box and tail-suspension test suggest normal levels of anxiety and lack of a "behavioural despair" phenotype in the knockout mice. Ncs-1 knockout and heterozygous knockout mice performed at wildtype levels in the Y-maze, suggesting intact spatial working memory. No genotype differences were revealed in tests of fear memory, whether measured $1 \mathrm{~h}$ or $24 \mathrm{~h}$ after conditioning, suggesting intact short-term and long-term fear memory in Ncs-1 knockout mice.

Conclusion: An initial behavioural screen suggests that global Ncs-1 knockout from birth does not have obvious impacts on locomotion, anxiety, levels of "despair," working memory, or fear memory in mice.

\section{2-41 Pathobiology of epithelioid sarcoma: mechanism of SMARCB1 inactivation}

Jamshidi F. ${ }^{1}$, McPherson A. ${ }^{2}, \mathrm{Ha}$ G. $^{2}$, Shah S..$^{2}$, Dickson B.C. ${ }^{3}$, Wunder J. 4,7, Andrulis I.L. 3,7, Huntsman D.G. ${ }^{5}$, Nielsen, T.O. ${ }^{6}$ ${ }^{1} \mathrm{MD} / \mathrm{PhD}$ Program, University of British Columbia, ${ }^{2} \mathrm{Com}-$ putational Cancer Biology, BC Cancer Agency, Vancouver, BC, Canada, ${ }^{3}$ Department of Pathology and Laboratory Medicine, Mount Sinai Hospital, ${ }^{4}$ Department of Surgery, Faculty of Medicine, University of Toronto, ON, Canada, ${ }^{5}$ Centre for Translational and Applied Genomics (CTAG), BC Cancer Agency, ${ }^{6}$ Department of Pathology and Laboratory Medicine, University of British Columbia, Vancouver, BC, Canada, ${ }^{7}$ Samule Lunenfeld Research Institute, Mount Sinai Hospital, Toronto, ON, Canada

Background: Epitheliod sarcoma (ES) is soft tissue malignancy of the extremities with the average age of presentation at $27(1)$. Although this tumour can manifest a deceptively non- aggressive gross appearance, it is highly metastatic, with a 10 year survival of $50 \%$ (2). There are currently no targeted drug therapies available.

The only established biologic feature of epithelioid sarcoma is lack of SMARCB1 expression, which is seen in $90 \%$ of cases (3). This protein is a known tumor suppressor characteristically inactivated in the pediatric malignant rhabdoid tumor of the kidney and its central nervous system counterpart (atypical teratoid rhabdoid tumor), wherein point mutations, truncations and copy number loss of SMARCB1 are common features. The silencing mechanism of SMARCB1 in epithelioid sarcoma is not established.

Purpose: To elucidate the mechanism of SMARCB1 inactivation in epithelioid sarcoma, for therapeutic exploitation.

Methods: Twenty-one cases were obtained via collaboration with the Cleveland Clinic, MD Anderson Cancer Centre and Mount Sinai Hospital in Toronto. Immunohistochemistry was carried out on 20 cases using the BAF47 antibody against SMARCB1. Sanger sequencing was carried out on all 21 cases including the 5' and 3'UTR and the 9 exons of SMARCB1. Illumina deep sequencing was completed on the transcriptomes of 6 cases and analyzed by deFuse (4). Fluorescent in situ hybridization (FISH) was carried out on 10 cases with probes hybridizing to the 5' and 3' ends of SMARCB1. Copy number analysis was done based on SNP Array 6.0 on 11 cases.

Results: Immunohistochemistry revealed loss of SMARCB1 expression in 16/20 cases. Copy number analysis rejected the reported idea (5) that homozygous deletion is the mechanism of SMARCB1 inactivation in ES. Furthermore, our sequencing results confirmed the previously reported finding of rare mutational events at the SMARCB1 locus in epithelioid sarco$\mathrm{ma}(6)$. However, deep sequencing of the transcriptome revealed 2 of 6 cases with rearrangements. FISH done on 1 of these 2 cases confirmed the result yet revealed the presence of non-rearranged copies as well. Only 1 additional case revealed genomic rearrangement by FISH. Our results suggest that ES is distinct from related pediatric malignancies exhibiting SMARCB1 inactivation in not having point mutations nor genome level deletions as the inactivating mechanism of SMARCB1. Instead, gene rearrangements are a novel mechanism likely contributing to SMARCB1 inactivation in this tumor and the contribution of epigenetic and/or posttranscriptional modifications are being actively pursued. 
Conclusions: Epithelioid sarcoma is a distinct biological entity with a unique mechanism of SMARCB1 inactivation. Rearrangements of the SMARCB1 transcript are seen in this tumor and epigenetic/post-transcriptional silencing are strong candidates.

\section{References:}

1. McPhee, M., McGrath, B.E., Zhang, P.,Driscoll D., Gibbs, J. and Peimer, C: Soft Tissue Sarcomas of the Hand. J of Hand Surgery, 24A: 1001-1007, 1999

2. Gary D. et al: Epithelioid sarcoma, An analysis of Fifty-One cases, Journal of bone and Joint Surgery (Am), 70-A: 862-870, 1988

3. Hornick JL, Dal Cin P, Fletcher CDM. Loss of INI1 expression is characteristic of both conventional and proximal-type epithelioid sarcoma. The American journal of surgical pathology [Internet]. 2009

4. McPherson A, Hormozdiari F, Zayed A, Giuliany R, Ha G, Sun MGF, et al. deFuse: An Algorithm for Gene Fusion Discovery in Tumor RNA-Seq Data. PLoS computational biology [Internet]. 2011

5. Modena, P. et al. SMARCB1/INI1 tumor suppressor gene is frequently inactivated in epithelioid sarcomas. Cancer Res. 65, 4012 4019, 2005.

6. Kohashi K, Izumi T, Oda Y, Yamamoto H, Tamiya S, Taguchi T, et al. Infrequent SMARCB1/INI1 gene alteration in epithelioid sarcoma: a useful tool in distinguishing epithelioid sarcoma from malignant rhabdoid tumor. Human pathology [Internet]. 2009

\section{2-42 TRPM2 is Implicated in Cellular Senescense and A $\beta$-Mediated Toxicity in Hippocampal Neurons}

Jillian C. Belrose ${ }^{1,2}$; Matthew Johnston ${ }^{1,3}$; Megan Chan ${ }^{1,3}$; Hongbin Li ${ }^{1}$; Michael F. Jackson ${ }^{1,3}$; John F. MacDonald ${ }^{1-3}$

${ }^{1}$ Robarts Research Institute, ${ }^{2}$ Depart. of Anatomy and Cell Biology, ${ }^{3}$ Depart. of Physiology and Pharmacology University of Western Ontario, London, ON, CAN

Background: The Transient Receptor Potential Melastatin 2 (TRPM2) channel is activated in response to oxidative stress, and plays a critical role in cell death in a variety of cell populations, including neurons. Alzheimer's disease is a progressive neurodegenerative disorder attributed to aging, oxidative stress, $A \beta$ oligomers, and a triad between hyperphosphorylated tau, Fyn kinase, and GluN2B containing NMDA receptors. Here, we assess whether TRPM2 currents are modulated by factors implicated in Alzheimer's disease including age, exposure to $A \beta$ oligomers, and Fyn kinase.
Methods: Primary cultured hippocampal neurons or HEK293 cells expressing TRPM2 were employed. In whole cell patch clamp recordings ADP-ribose, the intracellular ligand for TRPM2, was added to the patch electrode. Applications of NMDA or voltage-ramps were used to elicit a TRPM2 response in neurons. An MTT reduction assay was used to assess cell viability.

Results: As hippocampal neurons aged in vitro, TRPM2 currents increased due to a depletion of intracellular glutathione (GSH). GSH inhibited TRPM2 currents in HEK293 cells, producing a 3.5 fold shift in activation by ADP-ribose. We also demonstrate that Fyn kinase, a non-receptor tyrosine kinase recently implicated in Alzheimer's disease, co-immunoprecipitated with and phosphorylated TRPM2, and augmented TRPM2 currents in both HEK293-TRPM2 and neuronal cells. Furthermore, in cultured hippocampal neurons, treatment with A $1-42$ oligomers substantially enhanced TRPM2 responses. This was not observed in HEK293-TRPM2 cells, suggesting an indirect mechanism of TRPM2 channel activation by $A \beta$. Enhanced TRPM 2 currents following glutathione depletion, Fyn kinase application, and $A \beta$ exposure suggests that TRPM2 may play a role in Alzheimer's disease. Indeed, genetic deletion of TRPM2 attenuates the loss of cell viability mediated by $A \beta$ oligomer exposure in cultured hippocampal neurons. Preliminary results further suggest that knock-out of TRPM2 in an Alzheimer's model may improve performance in a spatial learning task.

Conclusions: Our results implicate TRPM2 in the calcium dysregulation and neurodegeneration associated with Alzheimer's disease, and suggest that pharmacological manipulation of the TRPM2 channel may have therapeutic potential.

\section{2-43 The role of macrophages in host cellular responses to Neisseria gonorrhoeae}

\section{Gordon G. McSheffrey; Scott D. Gray-Owen}

Department of Molecular Genetics, University of Toronto

Background: Neisseria gonorrhoeae is a human-restricted pathogen mainly associated with infection of the urogenital tract, gonorrhoea. Infection with N. gonorrhoeae can lead to serious complications such as pelvic inflammatory disease and sterility. N. gonorrhoeae causes over 90 million new infections per year and, recently, strains have been identified that are resistant to every known antibiotic. In order to survive within humans, the gonococcus has evolved sophisticated methods of 
evading the immune response. In particular, N. gonorrhoeae expresses an outer membrane protein called Opa, which binds to the host receptor, the Carcinoembryonic Antigen-related Cellular Adhesion Molecule-1 (CEACAM1). CEACAM1 is widely expressed on epithelial, endothelial, myeloid and lymphocyte cells. The Opa-CEACAM1 interaction allows the gonococcus to adhere to and invade epithelial and endothelial cells. Furthermore, the Opa-CEACAM1 interaction suppresses the activation of CD4+ T cells, an important mechanism by which $\mathrm{N}$. gonorrhoeae suppresses the immune response. Shockingly, there are very few studies examining the role of macrophages in gonococcal infection. Tissue resident macrophages constitute more than $2 \%$ of the resident cell population of the urogenital tract and are the first cell type encountered by the gonococcus after crossing the epithelial barrier. Macrophages play a crucial role in the immune response via pathogen recognition, killing and antigen presentation. In this study, we sought to examine the interaction of $\mathrm{N}$. gonorrhoeae with macrophages.

Methods: Using macrophages differentiated from monocytic cell lines, we examined the secretion of proinflammatory mediators by macrophages in response to infection with $\mathrm{N}$. gonorrhoeae.

Results: We observed a decrease in the secretion of IL-6, IL-8, and TNF $\alpha$ from macrophages infected with $N$. gonorrhoeae that expressed Opa and bound CEACAM1 compared to strains that did not.

Conclusions: The Opa-CEACAM1-dependent decrease in the secretion of proinflammatory mediators by macrophages suggests that $\mathrm{N}$. gonorrhoeae may deactivate macrophages and this contributes to gonococcal pathogenesis.

\section{2-44 The leading cause of cancer-related death in chil- dren is due to brain tumours}

\section{Xin (Kevin) Wang \\ $\mathrm{MD} / \mathrm{PhD}$ Candidate, University of Toronto}

The leading cause of cancer-related death in children is due to brain tumours, and medulloblastoma is the most common malignant paediatric brain tumour. Although advances in genetic technologies have enabled us to characterize these tumours at the molecular level, medulloblastoma pathogenesis is still poorly understood, and, of grievous importance, there are no currently approved targeted therapies. Recently, the Sleeping
Beauty (SB) transposon has been developed to take a functional genomic approach to study novel genes involved in cancer growth. The SB system utilizes a DNA element that can randomly mobilize through the genome when in the presence of an activating enzyme, transposase, ultimately driving tumorigenesis through overexpression of oncogenes and silencing of tumour suppressor genes. Our lab has previously developed a SB driven mouse model, where we were able to generate a $100 \%$ penetrance model of disseminated medulloblastoma. The current project looks to to develop an immune-competent in vivo animal model that will allow us to discriminate maintenance genes from initiation genes and passenger events, as these are the genes we must target in the design of novel therapeutics. We have designed and synthesized a novel transposon that contains the recognition sites for the $\mathrm{SB}$ transposase on the outside of the recognition sites for the PB transposase. In order to distinguish between maintenance and passenger events, we will induce $\mathrm{PB}$ transposition (using an inducible form of PB activated by tamoxifen) with administration of low and recurrent doses of tamoxifen. We observe that recurrent low-level PB-induced transposon re-mobilization in this model will result in an enrichment for maintenance events and a depletion of passenger events resulting in the discovery of new cancer genes.

\section{2-45 Do schizophrenics perceive spatial relationships differently from controls around the time of saccadic eye movements?}

\section{Alby Richard',2; Jan Churan'; Gillian O'Driscoll'; Debra Ti- tone $^{3}$; Christopher Pack ${ }^{2}$ \\ ${ }^{1}$ Leaders in Medicine/MD-Plus, Faculty of Medicine, Univer- sity of Calgary \\ ${ }^{2}$ Neurology and Neurosurgery, Montreal Neurological Insti- tute, McGill University \\ 3Department of Psychology, McGill University}

Background: In primates, inspection of a visual scene is typically interrupted by frequent gaze shifts, occurring at an average rate of 3-5 times per second. Perceptually, these gaze shifts are accompanied by a compression of visual space towards the saccade target, which may be attributed to an oculomotor, or corollary discharge (CD) signal, that transiently influences visual processing. Previous work has suggested this CD alters spatial processing around the time of saccades to explain the compression effect (Hamker et al., PLoS Comput Biol, 2008). 
Few studies, however, have examined the consequences of an impaired CD on perisaccadic visual perception.

While recent work has suggested that schizophrenic subjects lack a CD signal to cancel self-induced visual motion by smooth pursuit (Haarmeier et al., Curr Biol, 2005), it is not known whether they lack a CD signal for encoding saccadic eye movements. It is thus important to assess the performance of schizophrenics on perisaccadic localization, since without a CD these subjects should make fewer perisaccadic localization errors relative to controls.

Methods: Using a standard compression paradigm, we studied mislocalization in schizophrenic subjects and controls making horizontal $20^{\circ}$ eye saccades. Subjects were instructed to report the perceived position a briefly $(12 \mathrm{~ms})$ flashed vertical bar presented over a range of horizontal positions in a time-window \pm $200 \mathrm{~ms}$ around saccade onset.
Results: We found a global compression of visual space for both schizophrenic and control subjects that depended on the time at which the vertical bar was presented relative saccade onset. Importantly, a more detailed analysis of individual bars revealed differences in both the magnitude and temporal profile of compression between the two groups, consistent with an altered CD in schizophrenics.

Conclusions: These results, which could be captured by our previous model of compression (Richard et al., J Neurosci, 2009; J Vis, 2011), provide new information about perceptual processing in schizophrenic populations. Moreover, the present findings expand on what is known about the extra-retinal mechanisms that maintain perceptual constancy across saccades, while also providing further insight into the neurological basis of specific positive symptoms.

Funding: CIHR, Alfred Sloan Foundation 